\title{
3. Die arbeitsrechtlichen Veränderungen
}

\section{Gesetzbuch der Arbeit}

Die SED hatte auf der II. Parteikonferenz 1952 den Beschluß gefaßt, ein Arbeitsgesetzbuch ausarbeiten zu lassen 1 . Bereits zwei Jahre zuvor war das Gesetz der Arbeit in Kraft getreten, das nunmehr der forcierten Umgestaltung von Staat, Wirtschaft und Gesellschaft, welche die II. Parteikonferenz einläutete, angepaßt werden sollte. Eine Parteikommission erhielt den Auftrag, einen entsprechenden Entwurf auszuarbeiten und anschließend der SED-Führung vorzulegen. Neben dem Arbeitsgesetzbuch war noch ein Zivil- sowie ein neues Strafgesetzbuch vorgesehen. Vorsitzender der Kommission war Prof. Dr. Heinz Such ${ }^{2}$ (Universität Leipzig); die unmittelbare Verbindung zum ZK der SED und die Organisation der Arbeit der Kommission übernahm die Justizministerin Hilde Benjamin ${ }^{3}$. Der Volksaufstand vom 17. Juni 1953 und der „Neue Kurs“ verzögerten vermutlich die Tätigkeit dieses Gremiums. Dennoch ging die Vorbereitung eines neuen Arbeitsgesetzbuches "mit ausdrücklicher Zustimmung" der SED-Führung weiter4: Am 2. Juli 1954 konnte der ZK-Abteilung Staatliche Verwaltung ein erster Entwurf übergeben werden, an dem Vertreter des FDGB-Bundesvorstandes und des Ministeriums für Arbeit mitgewirkt hatten. Um die steckengebliebene Gesetzesausarbeitung voranzutreiben, schlug Benjamin vor, in der ersten Julihälfte eine mehrtägige Konferenz durchzuführen, auf der der Entwurf und das weitere Vorgehen beraten werden sollten. Basierend auf den gewonnenen Ergebnissen dieser Konferenz sollten dann der „bisherige“ Entwurf sowie die Änderungsvorschläge beim FDGB-Bundesvorstand und beim Arbeitsministerium nochmals erörtert werden. Die Leitungsgremien des FDGB (Sekretariat des Bundesvorstandes) und des Arbeitsministerium (Kollegium) hatten anschließend - so die Überlegung der Justizministerin - einen Beschluß darüber zu fassen, der wiederum Richtschnur für die Kommission bei der Fertigstellung des Entwurfs sein sollte. Nach Abschluß dieses Abstimmungsverfahrens war die Vorlage beim Politbüro einzureichen.

Mit diesem Vorschlag konnte sich die Justizministerin offenbar nicht durchsetzen. Das Sekretariat des FDGB-Bundesvorstandes beriet erst im November 1954 über einen entsprechenden Gesetzesentwurf, der interessanterweise gesamtdeutsche Ambitionen erkennen ließ und vor allem den Versuch unternahm, soziale Grundrechte zu fixieren und inhaltlich auszufüllen ${ }^{5}$. Diese waren zwar in anderen arbeitsrechtlichen Bestimmungen bereits enthalten, sollten aber nochmals in einem Gesetzeswerk zusammengefaßt werden. Zu diesen Grundrechten sollten das

1 Protokoll der II. Parteikonferenz der SED, S. 493.

2 Keine Angaben zu Suchs Leitungsfunktion innerhalb dieser Kommission sowie zu ihrer inhaltlichen Tätigkeit enthält die rechtswissenschaftliche Biographie von Stiebitz, Heinz Such.

3 Die beiden kürzlich erschienenen Biographien gehen nur auf Benjamins Einfluß bei der Ausarbeitung des Strafgesetzbuches sowie des Familiengesetzbuches ein. Dagegen wird das Arbeitsgesetzbuch überhaupt nicht thematisiert. Vgl. Feth, Hilde Benjamin; Brentzel, Die Machtfrau.

4 BAB, DQ 2/536, Justizministerin Hilde Benjamin am 27.6. 1955 an Arbeitsminister Macher.

5 SAPMO, DY 34/24288, Protokoll der Sitzung des Sekretariats des FDGB-Bundesvorstandes vom 22. 11. 1954. Die Sekretariatsvorlage vom 16.11. 1954 stammte vom Büro für deutsche Gewerkschaftseinheit beim FDGB-Bundesvorstand. 
Recht auf Arbeit, das Recht auf Entlohnung, das Recht auf Urlaub und das Recht auf Schutz der Arbeitskraft gehören ${ }^{6}$. Der Gewerkschaftsentwurf wurde nochmals überarbeitet und inhaltlich erweitert?: So kamen eigene Abschnitte zum Koalitions-, Streik- und Mitbestimmungsrecht, zur Berufsausbildung, zum Kündigungsrecht, zur Arbeitszeitregelung, zur Gesundheitsfürsorge sowie allgemeine sozialversicherungsrechtliche Bestimmungen hinzu. Das Sekretariat des FDGBBundesvorstandes konnte sich zu einer raschen Verabschiedung der gewerkschaftseigenen Vorlage nicht durchringen. Statt dessen befaßte sich das Führungsgremium mehrfach mit dem Arbeitsgesetzbuch und signalisierte zwar grundsätzliche Zustimmung, reichte aber den Entwurf immer wieder an die entsprechenden Abteilungen des FDGB-Bundesvorstandes zurück. Vermutlich wollte die FDGBFührung ein vorschnelles Handeln vermeiden und zunächst einmal die Entscheidungsfindung innerhalb der SED-Führung abwarten. Dieses Verhalten bestätigte indirekt die untergeordnete Position, welche die Gewerkschaften mittlerweile aus eigenem Antrieb heraus gegenüber der Hegemonialpartei einnahmen. Ende 1954 bestätigte das Sekretariat des FDGB-Bundesvorstandes die eingereichte Vorlage „im Prinzip“8. Rund acht Monate später stand das Thema erneut auf der Tagesordnung: Dabei wurden die "Grundprinzipien“ eines erheblich erweiterten Entwurfes, der vermutlich von der Kommission zur Ausarbeitung des Arbeitsgesetzbuches stammte, erneut bestätigt und eine weitere Überarbeitung angeregt ${ }^{9}$. Damit verzichtete der FDGB darauf, einen eigenen Entwurf in die Debatte über die Gestaltung eines neuen Arbeitsgesetzbuches einzubringen. Die ZK-Abteilungen Arbeit, Sozial- und Gesundheitswesen und Staatliche Organe überreichten Walter Ulbricht am 18. Oktober 1955 einen zweiten Entwurf $1{ }^{10}$, der allerdings rasch fallengelassen wurde. Das Politbüro setzte nämlich am 28. August 1956 eine neue Kommission ein, die vermutlich im November eine neue Gliederung des Arbeitsgesetzbuches präsentierte ${ }^{11}$.

Obwohl zwischen den beteiligten Stellen (ZK-Abteilungen, Arbeitsministerium, FDGB) Meinungsunterschiede oder Interessengegensätze nicht offen zu erkennen waren, ruhten die Arbeiten am Gesetzesvorhaben für ungefähr zwei Jahre. Erst im Herbst 1958 griff die ZK-Abteilung Gewerkschaften, Sozial- und Gesundheitswesen - nach Absprache mit dem Politbüro - das eingeschlafene Projekt wieder auf und legte eine Grobgliederung für das geplante Arbeitsgesetzbuch vor $^{12}$. Die ursprüngliche eingesetzte Kommission hatte offenbar aufgehört zu existieren, so daß nunmehr ein neues Gremium eingerichtet werden mußte, dem wiederum Vertreter des ZK-Apparates, des Komitees für Arbeit und Löhne, der Gewerkschaften und einiger arbeitsrechtlicher Institute angehören sollten. In der

6 Ebenda, Sekretariatsvorlage vom 16.11. 1954.

7 SAPMO, DY 34/24293, Entwurf (Gesetz der Arbeit) des Büros für deutsche Gewerkschaftseinheit vom 23. 12. 1954.

8 Ebenda, Protokoll der Sekretariatssitzung des FDGB-Bundesvorstandes vom 27. 12. 1954.

- SAPMO, DY 34/24330, Protokoll der Sekretariatssitzung des FDGB-Bundesvorstandes vom 17. 8. 1955. Das Protokoll enthält eine umfangreiche Liste mit Änderungsvorschlägen, die von der Abt. Arbeitsrecht des FDGB-Bundesvorstandes ausgearbeitet wurden.

10 SAPMO, DY 30/IV 2/6.11/60, Bl. 2-4, hier Bl. 2f., Notiz der Abt. Arbeit, Sozial- und Gesundheitswesen (o.D.).

11 Ebenda, Bl. 3.

12 SAPMO, DY 30/IV 2/13/46, Bl. 171-185, Vorlage vom 26. 10. 1958. 
Erläuterung zur Grobgliederung machte die ZK-Abteilung den Funktionswandel deutlich, den die SED dem Arbeitsrecht und ganz besonders dem geplanten Arbeitsgesetzbuch zukommen lassen wollte: „Das Arbeitsgesetzbuch muß ein Mittel des volksdemokratischen Staates werden, um die sozialistischen Produktionsund Arbeitsverhältnisse zu festigen und die sozialistische Arbeit planmäßig und bewußt zu organisieren. Es soll die Entwicklung des sozialistischen Bewußtseins fördern, die Erziehung der Werktätigen zu einer neuen Arbeitsmoral unterstützen und zur Überwindung der Reste bürgerlichen Denkens beitragen." 13 Darüber hinaus durfte - drei Monate nach dem V. Parteitag - ein Hinweis auf die Überlegenheit der DDR gegenüber der Bundesrepublik und die deutschlandpolitischen Absichten nicht fehlen: „Das sozialistische Arbeitsgesetzbuch zeigt die Überlegenheit der staatlichen und rechtlichen Ordnung der DDR gegenüber der überlebten, zum Untergang verurteilten kapitalistischen Ordnung und unterstützt damit den Kampf der deutschen Arbeiterklasse gegen das imperialistische Regime in Westdeutschland und für die nationale Wiedervereinigung Deutschlands zu einem friedliebenden, demokratischen Staat." Insofern kann die Wiederaufnahme der Arbeiten an dem Arbeitsgesetzbuch 1958, die lange Zeit liegengeblieben waren, durchaus mit den allgemeinen ideologischen und ökonomischen Offensiven der SED-Führung in Zusammenhang gebracht werden. Im einzelnen sollte das Gesetzeswerk das Arbeitsrecht vereinfachen und die „verstreuten und unübersichtlichen" arbeitsrechtlichen Bestimmungen zusammenfassen. Das neue Arbeitsgesetzbuch sollte übersichtlich gegliedert und ,in einer jedem Arbeiter verständlichen Sprache gehalten sein"14. Damit war in erster Linie ein Rahmengesetz bzw. eine Kodifikation des bestehenden Arbeitsrechts vorgesehen; Detailfragen sollten dagegen in „Kollektivvereinbarungen“ geregelt werden. Die ZK-Abteilung Staat und Recht war mit der vorgelegten Disposition grundsätzlich einverstanden, wies aber gleichzeitig darauf hin, daß sich „bei der weiteren Ausarbeitung eine Reihe von Problemen ergeben [werden], deren Einzelheiten jetzt noch nicht übersehen werden können" 15 .

Erneut war es die ZK-Abteilung Gewerkschaften, Sozial- und Gesundheitswesen, die die Initiative ergriff und am 2. Dezember 1958 eine Vorlage für das Politbüro erstellte' ${ }^{16}$. Diese enthielt nochmals die bereits ausgearbeitete Grobgliederung und schlug einen Terminplan vor. Demnach sollte der Entwurf eines Arbeitsgesetzbuches bis zum 30. Juni 1959 dem Politbüro unterbreitet werden. Das Politbüro bestätigte erst auf seiner Sitzung am 20. Januar 1959 die vorgelegte Grundlinie und Grobgliederung zum Arbeitsgesetzbuch ${ }^{17}$. Damit trat die Ausarbeitung des Gesetzeswerkes in eine entscheidende Phase: Nunmehr konnten die in der Kommission versammelten Vertreter der ZK-Abteilungen, des Komitees für Arbeit und Löhne sowie des FDGB-Bundesvorstandes mit ihrer Arbeit fort-

13 Ebenda, Bl. 177.

14 Ebenda, Bl. 178. Das Sekretariat des FDGB-Bundesvorstandes ging auf die Grobgliederung und Erläuterung zum Arbeitsgesetzbuch inhaltlich nicht ein, sondern befaßte sich mit Verfahrens- und Personalfragen (Zusammensetzung der Kommission). Vgl. SAPMO, DY 34/24507, Protokoll der Sitzung des Sekretariats des FDGB-Bundesvorstandes am 1. 11. 1958.

is SAPMO, DY 30/IV 2/13/46, Bl. 120, Vermerk Grünebergs für Verner vom 12. 11. 1958.

16 SAPMO, DY 30/IV 2/6.11/60, Bl. 5-20.

17 SAPMO, DY 30/J IV 2/2/628, Bl. 7 und 26-41. 
fahren und die Detailbestimmungen festlegen. Entsprechend der allgemeinpolitischen Entwicklung, welche die SED-Führung auf dem V. Parteitag entworfen hatte, war das Arbeitsgesetzbuch nur für eine Übergangszeit vorgesehen ${ }^{18}$. Hintergrund dafür bildete das weitere Fortbestehen unterschiedlicher Eigentumsformen in der Wirtschaft: Neben der sozialistischen, d.h. volkseigenen und genossenschaftlichen, bestand nach wie vor noch die private Wirtschaftsform. Bei der Ausarbeitung des Gesetzentwurfes wurde auch die für Rechtsfragen zuständige ZK-Abteilung beratend hinzugezogen. Diese hatte die Beratungen einer eigens für die Fertigstellung von zwei großen Gesetzesvorhaben (Arbeitsgesetzbuch, $\mathrm{Fa}$ miliengesetzbuch) einberufenen Kommission für Staats- und Rechtswissenschaft geleitet ${ }^{19}$. Offensichtlich war geplant, das Arbeitsgesetzbuch dem Politbüro ungefähr Mitte August 1959 vorzulegen ${ }^{20}$. Die Gewerkschaften drängten auf eine rasche Verabschiedung des Entwurfs und konnten die ZK-Abteilung Gewerkschaften und Sozialpolitik für ihre zeitlichen Vorstellungen gewinnen. Diese wandte sich direkt an Walter Ulbricht und bat um die „Klärung dieser und einiger anderer Fragen, die damit im Zusammenhang stehen" 21 .

Für die Bezirksverwaltungen bzw. die dortigen Abteilungen für Arbeit und Berufsausbildung besaß vor allem Artikel 77 des Entwurfes vom 15. Juli 1959 besondere Bedeutung. Dieser schrieb nochmals das Recht auf Arbeit fest. Die Begründung zu diesem Gesetzesartikel enthielt die arbeitsmarktpolitische Zielsetzung: „Die Regelungen bezwecken, das Ausscheiden von Werktätigen aus dem Betriebskollektiv auf das gesellschaftlich notwendige Maß zu beschränken und die Fluktuation der Arbeitskräfte zu bekämpfen. “22 Die bestehenden Freiräume der Betriebe, Beschäftigte zu entlassen, sollten „erheblich“ eingeengt werden. So sei die fristgemäße Kündigung nunmehr eine „wirtschaftlich-organisatorische Maßnahme“, während die fristlose Entlassung die "härteste Disziplinarstrafe“ darstelle, die „nur im äußersten Fall angewandt werden darf und dabei dem Schutz des sozialistischen Betriebskollektivs dient". Da diese Auslegung weitgehend unscharf blieb, besaßen die Betriebe auch weiterhin die Möglichkeit, sich von Arbeitern oder Angestellten zu trennen. Sehr viel konkreter wurden dagegen die Aussagen zum Aufhebungsvertrag, der bisher unbeschränkt zulässig war, mit dem geplanten Arbeitsgesetz aber auf bestimmte Ausnahmesituationen zu begrenzen war. Auch hier bot allerdings der Zusatz, „wenn er [der Aufhebungsvertrag] gesellschaftlich gerechtfertigt ist", relativ großen Interpretationsspielraum. Nach Auffassung der Staatlichen Plankommission sollte das bereits zuvor fixierte Recht auf Arbeit mit der „Ehrenpflicht“ jedes Bürgers gekoppelt werden, „seine Arbeitskraft dem sozialistischen Aufbau zur Verfügung zu stellen"23. Somit fand die Arbeitspflicht - zumindest indirekt - Einzug in die arbeitsrechtlichen Regelungen. Nicht alle Abteilungen der Staatlichen Plankommission waren bei den Vor-

18 BAB, DQ 3/486, Bericht des Komitees für Arbeit und Löhne vom 20. 2. 1959.

19 SAPMO, DY 30/IV 2/13/46, Bl. 121-145, Protokoll der Sitzung der Kommission für Staats- und Rechtswissenschaft am 16.3.1959.

20 SAPMO, DY 30/IV 2/6.11/60, Bl. 21, Notiz der ZK-Abt. Gewerkschaften und Sozialpolitik für Walter Ulbricht vom 13. 7. 1959.

21 Ebenda.

22 BAB, DE 1/9051, Bl. 28 f., 1. Entwurf des Arbeitsgesetzbuches vom 15. 7. 1959.

23 $\mathrm{BAB}, \mathrm{DE}$ 1/9055, Bl. 12, Bemerkungen vom 21. 7. 1959 zum 1. Entwurf des Arbeitsgesetzbuches. 
arbeiten am Arbeitsgesetzbuch von Anfang an beteiligt: So erhielt etwa die Abteilung Arbeitskräfte den 1. Entwurf erst nach seiner Fertigstellung. Dabei wies die Abteilung darauf hin, daß die die Kündigungsfragen betreffenden Paragraphen keinerlei Angaben enthielten über Kündigungen von seiten der Beschäftigten ${ }^{24}$. Sie schlug in dem Zusammenhang vor, die Durchführung von "gründliche[n] Aussprachen" zwischen dem betroffenen Beschäftigten und den gewerkschaftlichen Organen des Betriebes vorzuschreiben, um ein Ansteigen der beruflichen Fluktuation zu verhindern. Oberstes Ziel war letztlich die Heranbildung und langfristige Absicherung von betrieblichen Stammbelegschaften. Bei der Ausarbeitung des 2. Entwurfes im Sommer 1960 wurde dieser Vorstoß partiell berücksichtigt: „Im Interesse der Steigerung der Arbeitsproduktivität und der Schaffung fester Betriebskollektive haben die Betriebsleiter darauf hinzuwirken, daß jedes gesellschaftlich unnötige Ausscheiden aus dem Betriebskollektiv vermieden wird." 25

Das Politbüro stimmte dem überarbeiteten Entwurf Ende Oktober 1960 „als Grundlage für die öffentliche Diskussion" $\mathrm{zu}^{26}$. Eine Kommission erhielt den Auftrag, die Schlußredaktion zu übernehmen und den Entwurf anschließend dem Ministerrat zur Beratung vorzulegen. Inhaltliche Veränderungen ergaben sich für die Arbeitskräfteplanung und -lenkung in erster Linie durch die Wiedereinführung der Arbeitseinweisung, die nunmehr als Zuweisung einer anderen Arbeit bezeichnet und an zwei Voraussetzungen gebunden wurde. Der Einsatz dieses Zwangsinstruments war möglich, wenn „das zur Sicherung der Planerfüllung bzw. der betrieblichen Aufgaben erforderlich ist, obwohl der Werktätige in seinem Arbeitsbereich tätig sein könnte, oder in seinem Arbeitsbereich die sachlichen Voraussetzungen zur Arbeitsleistung fehlen"27. Gleichzeitig wurden zeitliche Fristen für die Arbeitszuweisung eingeführt, die zwischen einem und sechs Monaten lagen. Damit griff das geplante Arbeitsgesetzbuch auf Bestimmungen der zentralen Verordnung vom 2. Juni 1948 zurück, die aber für die betroffenen Beschäftigten sozialpolitisch besser abgefedert wurden. Das Führungsgremium der SED beschloß des weiteren einen Plan „zur Organisierung der Diskussion“ über das Arbeitsgesetzbuch, der an die 1. Sekretäre der Bezirks- und Kreisleitungen der Hegemonialpartei weitergeleitet wurde und umfangreiche propagandistische Aktivitäten veranlassen sollte28.

Die SED-Führung plante sehr sorgfältig die Verabschiedung des Arbeitsgesetzbuches. Nachdem das Politbüro seine Zustimmung gegeben hatte, erhielt das Präsidium des Ministerrates den fertiggestellten Gesetzentwurf ${ }^{29}$. Erst jetzt konnten die Minister, die zuvor nicht konsultiert worden waren, eigene Verbesserungsvorschläge einreichen. Während ungefähr zeitgleich die Vorbereitungen für die Veröffentlichung des Gesetzes anliefen, bei denen vor allem der FDGB eingeschaltet

${ }^{24}$ Ebenda, Bl. 17, Leiter der Abt. Arbeitskräfte (Duscheck) am 10. 9. 1959 an den Leiter der Rechtsstelle (Kothe).

$25 \mathrm{BAB}, \mathrm{DE} 1 / 9052, \mathrm{Bl} .83,2$. Entwurf des Arbeitsgesetzbuches vom 17. 7. 1960 ( $\$ 166$ ).

26 SAPMO, DY 30/J IV 2/2/731, Bl. 3, Protokoll der Sitzung des Politbüros am 25. 10. 1960.

27 Ebenda, Bl. 17, Entwurf des Arbeitsgesetzbuches ( $\$ 22$, Abs. 1).

28 Ebenda, Bl. 49-58.

29 BAB, DC 20 I/4-411, Bl. 104 f., 1. Sekretär des ZK der SED, Walter Ulbricht, am 31. 10. 1960 an den Vorsitzenden des Ministerrates der DDR, Otto Grotewohl. 
wurde ${ }^{30}$, trafen zwischen dem 9. und 11. November die Stellungnahmen der einzelnen Ressorts ein. Bei einem Vergleich der einzelnen Entwürfe fällt auf, daß im Zuge der Überarbeitung alle Bestimmungen gestrichen worden waren, die finanzielle Mehraufwendungen zur Folge gehabt hätten. Darauf hatte das Ministerium der Finanzen hingewiesen, das an der letzten Fassung des Entwurfs nur noch kleinere, d. h. keine substantiellen Änderungen vornehmen wollte ${ }^{31}$. Der Minister für Handel und Versorgung erteilte zwar seine prinzipielle Zustimmung zum vorliegenden Gesetzeswerk, übte allerdings auch indirekt Kritik an dem langwierigen Verfahren: „Die Durchsicht erweckt den Anschein, als wenn nicht alle Bestimmungen in dem jetzt lange zurückliegenden Zeitraum der Bearbeitung sorgfältig aufeinander abgestimmt sind."32 Folgende Ministerien bzw. staatliche Organe reichten zustimmende Stellungnahmen ein, die jedoch häufig mit Detailvorschlägen verknüpft waren: das Ministerium für Nationale Verteidigung ${ }^{33}$, das Ministerium für Kultur ${ }^{34}$, das Ministerium für Post- und Fernmeldewesen ${ }^{35}$, das Ministerium für Volksbildung ${ }^{36}$, die Staatliche Plankommission ${ }^{37}$ und das Ministerium für Bauwesen ${ }^{38}$. Bereits am 1. November 1960 stimmte das Präsidium des Ministerrates dem Entwurf des Arbeitsgesetzbuches zuํ.

Mitte Dezember 1960 erstellte das Ministerium für Staatssicherheit einen Bericht, der die Reaktionen der Bevölkerung auf den bekanntgewordenen Entwurf des Arbeitsgesetzbuches zu erfassen versuchte und ein zwiespältiges Gesamtbild vermittelte ${ }^{40}$. So wurde einerseits betont, daß die bisher erfaßten Diskussionen auf Betriebsversammlungen oder Veranstaltungen der Gewerkschaften „zum größten Teil zustimmende Erklärungen [beinhalteten], die auch teilweise ein richtiges Verständnis der politischen Bedeutung des Gesetzeswerkes erkennen " ließen ${ }^{41}$. Vor allem die erneute Festschreibung des Rechts auf Arbeit sei von der überwiegenden Mehrheit der Bevölkerung positiv aufgenommen worden. Andererseits gelangte das MfS zum Ergebnis, daß der Verlauf der eingeleiteten Popularisierungsmaßnahmen, die der FDGB übernommen hatte, „keineswegs den Erfordernissen entsprechen " würde ${ }^{42}$. Daher verwunderte es nicht, daß sich die Kritik vor allem gegen die Gewerkschaftsfunktionäre richtete, denen vorgehalten wurde, zu wenig Eigeninitiative entwickelt und nur auf die Anleitung durch übergeordnete Organe gewartet zu haben. In zahlreichen Betrieben hätten noch keine Aussprachen mit den Belegschaftsmitgliedern stattgefunden; darüber hinaus sei auf den bisherigen

30 Vgl. SAPMO, DY 30/J IV 2/3/711, Bl. 3, Protokoll der Sitzung des Sekretariats des ZK vom 7. 11. 1960.

31 BAB, DC 20 I/4-411, Bl. 109-112, Stellungnahme des Ministeriums der Finanzen vom 9. 11. 1960.

32 BAB, DC 20 I/4-411, Bl. 114 f., hier Bl. 115, Minister für Handel und Versorgung am 10. 11. 1960 an den Leiter des Sekretariats des Ministerrates, Staatssekretär Jendretzky.

33 Ebenda, Bl. $116 f$.

34 Ebenda, B1. 118.

35 Ebenda, Bl. 119.

36 Ebenda, Bl. 120-122.

37 Ebenda, Bl. 123-128.

38 Ebenda, Bl. 129-131.

39 BAB, DC 20 I/4-409, Bl. 9, Protokoll der Sitzung des Präsidiums des Ministerrates am 10.11. 1960.

40 BStU, MfS, ZAIG, 281, Bl. 1-7, Bericht vom 15. 12. 1960.

41 Ebenda, Bl. 2.

42 Ebenda, Bl. 1. 
Veranstaltungen die politische Bedeutung des Arbeitsgesetzbuches „ungenügend herausgearbeitet" worden. Außerdem kritisierte der Bericht, daß Mitglieder von FDGB-Kreisvorständen oder Betriebsgewerkschaftsleitungen häufig „nur vom arbeitsrechtlichen Standpunkt aus über die ,Rechte der Werktätigen diskutier[en], während die sich aus dem Entwurf ergebenden Verpflichtungen übergangen oder ungenügend geklärt" würden ${ }^{43}$. Nach Einschätzung des MfS hatten vor allem die Bestimmungen zur Arbeitszuweisung negative Auswirkungen auf die politische Stimmungslage in der DDR. Anschließend listete der Bericht einige Änderungsvorschläge auf, die auf den öffentlichen Veranstaltungen zum Arbeitsgesetzbuch vorgebracht worden waren: Dazu zählte unter anderem die Forderung, den bestehenden Gesetzestext dahingehend zu ergänzen, daß die zugewiesene Arbeit „zumutbar sein und dem Gesundheitszustand des jeweiligen Kollegen entsprechen muß “44. Abschließend führte der Stimmungsbericht des Ministeriums für Staatssicherheit Äußerungen von einzelnen, namentlich nicht kenntlich gemachten Teilnehmern dieser Versammlungen auf, welche vor allem den Zwangscharakter des neuen Gesetzes betonten: „Das Arbeitsgesetz ist ein Notstandsgesetz, da jeder entsprechend den Erfordernissen dort eingesetzt werden kann, wo er eben gebraucht wird.“45 Oder: „Diese Paragraphen [zur Arbeitszuweisung] kommen einer Dienstverpflichtung gleich. Es fehlt nur noch der Gummiknüppel.“ Andere wiesen sogar auf die Parallelität zu Maßnahmen des nationalsozialistischen Regimes hin: „Das neue Arbeitsgesetz kommt der Dienstverpflichtung und dem Arbeitsdienst der Nazizeit gleich. Es ist ein Gesetz der modernen Sklaverei." Das Ministerium machte jedoch nicht die inhaltliche Form oder einzelne gesetzliche Bestimmungen des Arbeitsgesetzbuches für die prekäre Stimmungslage in der Bevölkerung verantwortlich, sondern die mangelhafte Vorbereitung und Durchführung der Propagandamaßnahmen von seiten der Gewerkschaften.

Ungeachtet dieser Bedenken ${ }^{46}$ schritt die Fertigstellung des Arbeitsgesetzbuches nunmehr zügig voran. Nachdem bereits im November 1960 einige Ministerien ihre Änderungswünsche eingebracht hatten, folgten am 30. März 1961 das Ministerium für Gesundheitswesen ${ }^{47}$, das Ministerium für Verkehrswesen ${ }^{48}$ sowie das Staatssekretariat für das Hoch- und Fachschulwesen ${ }^{49}$. Grundsätzliche Einwände wurden von den beteiligten Ressorts jedoch nicht mehr vorgetragen, so daß das Politbüro die Beratung in der Volkskammer auf den 12. April festsetzen

43 Ebenda, Bl. $1 \mathrm{f}$.

44 Ebenda, Bl. 5.

45 Ebenda, Bl. 7.

46 In einer umfangreichen Stellungnahme setzte sich der Deutsche Gewerkschaftsbund (DGB) kritisch mit dem Arbeitsgesetzbuch auseinander und betonte vor allem den Zwangscharakter, den Verlust von sozialpolitischen Errungenschaften (Streikrecht) sowie die Funktionalisierung des Arbeitsrechts für wirtschaftspolitische Vorstellungen der SED-Führung. Vgl. BAB, DQ 3/861, Stellungnahme des DGB vom 3. 3. 1961. Zurückhaltende Kritik kam von Vertretern der evangelischen Kirche in der DDR. So bemängelte etwa Bischof Krummacher die vorrangige Zielsetzung des Gesetzentwurfes (Steigerung der Arbeitsproduktivität) und die Wiedereinführung der Arbeitszuweisung. Vgl. BAB, DQ 3/494, Bischof Krummacher am 13.3. 1961 an den Staatssekretär für Kirchenfragen (Seigewasser).

47 BAB, DC 20 I/4-442, B1. 82-84.

48 Ebenda, Bl. 72-74.

49 Ebenda, Bl. 75. 
konnte ${ }^{50}$. Bei der inhaltlichen Überarbeitung wurden die Bestimmungen zur Arbeitszuweisung letztlich entschärft: Zuweisungen, nunmehr euphemistisch als "vorübergehende Übertragung einer anderen Arbeit" bezeichnet, waren bei einem Zeitraum über einen Monat hinaus nur mit Zustimmung des Betroffenen zulässig51. Mit der Veröffentlichung des Arbeitsgesetzbuches im DDR-Gesetzblatt hatte eine jahrelange Diskussion über eine erneute Kodifizierung des Arbeitsrechtes ihren Abschluß gefunden. Mitte Juni 1961verabschiedete das Sekretariat des ZK schließlich noch einen Maßnahmenkatalog „zur Erläuterung und Durchführung" des Gesetzbuches der Arbeit ${ }^{52}$. Dabei sollten der FDGB, die Industriegewerkschaften und Gewerkschaften als „Hauptorganisator" auftreten ${ }^{53}$. Das Gesetzbuch der Arbeit stellte - wie bereits erwähnt - eine Zusammenfassung geltender arbeitsrechtlicher Bestimmungen dar und hatte kaum Auswirkungen auf die Arbeitskräftelenkung. Von entscheidender Bedeutung war die Tatsache, daß bei der Ausarbeitung eine Rückkehr zu Zwangseinweisungen, wie sie noch bis ungefähr 1949 des öfteren praktiziert worden waren, ausgeschlossen wurde. Einen ähnlichen Verlauf nahm im übrigen auch die Debatte über die Verordnung zur Verbesserung der Arbeitskräftelenkung und Berufsberatung, der wir uns im folgenden Abschnitt zuwenden wollen.

\section{Verordnung zur Verbesserung der Arbeitskräftelenkung und Berufsberatung}

Das Ministerium für Arbeit äußerte erstmals im Spätsommer 1953 grundsätzliche Zweifel an der Wirksamkeit der Verordnung über die Aufgaben der Arbeitsverwaltungen und über die Lenkung der Arbeitskräfte vom 12. Juli 1951. Die Neustrukturierung sowie die inhaltliche Zurückdrängung der Arbeitsverwaltung, die zunächst als Fortschritt angesehen wurde, geriet nunmehr ins Visier der Kritik: Durch diese Verordnung sei den Abteilungen für Arbeit und Berufsausbildung die Möglichkeit genommen worden, „unmittelbar auf die Lenkung und Verteilung der Arbeitskräfte Einfluß zu nehmen" 54 . Darüber hinaus wurde die gewachsene Eigenständigkeit der Betriebe bei der Anwerbung von Arbeitskräften durch das Ministerium für Arbeit mittlerweile als „nachteilig“ empfunden. Das Ministerium gelangte zum Ergebnis, daß die Betriebsleitungen „[z]um größten Teil“ die Beschäftigten entlassen würden, „die ohnehin schwer in den Arbeitsprozeß [sic] untergebracht werden können“. Von dieser Entwicklung waren vor allem ältere Frauen und Männer, alleinstehende Frauen, Jugendliche und Schwerbeschädigte betroffen ${ }^{55}$. Diese als schwer vermittelbar eingestuften Personengruppen ließen im ersten Halbjahr 1953 die Erwerbslosenzahlen zeitweilig etwas ansteigen. Nach

50 SAPMO, DY 30/J IV 2/2/758, Bl. 3, Protokoll der Sitzung des Politbüros am 5. 4. 1961; SAPMO, DY 30/J IV 2/2/759, Bl. 11, Protokoll der Sitzung des Politbüros am 11.4. 1961.

51 Gesetzbuch der Arbeit der DDR vom 12. 4. 1961 ( $\$ 25$, Abs. 1), in: Gesetzblatt der DDR 1961, Teil I, S. 27-49, hier S. 33.

52 SAPMO, DY 30/J IV 2/3/743, Bl. 2 und Bl. 28-32, Protokoll der Sitzung des Sekretariats des ZK am 15.6. 1961.

53 Ebenda, Bl. 28.

5+ $\mathrm{BAB}, \mathrm{DQ} 2 / 1707$, Begründung des Ministeriums für Arbeit vom 9.9. 1953 zur Verordnung über die Lenkung der Arbeitskräfte, S. 1.

55 Ebenda, S. 2. 
Ansicht des Ministeriums für Arbeit war der Anteil der genannten Beschäftigtengruppen an der Gesamtbeschäftigtenzahl "prozentual so gering“, daß von einer Belastung der Betriebe keine Rede sein konnte. Die Kritik an der zentralen Verordnung von 1951, die de facto bis 1961 Bestand haben sollte, war jedoch nicht nur eine Konsequenz der realistischen Einschätzung durch das Arbeitsministerium, sondern hing vermutlich auch mit dem politischen Kurswechsel und der Proklamierung des „Neuen Kurses“ zumindest indirekt zusammen. Nun rückte das bis dahin immer noch nicht erreichte Ziel - die vollständige Beseitigung der Arbeitslosigkeit - wieder verstärkt in den Mittelpunkt der Tätigkeit der Arbeitsverwaltung. Während zuvor davon ausgegangen worden war, daß der massive Auf- und Ausbau der Schwerindustrie ein Absinken der Erwerbslosenzahlen automatisch mit sich bringen würde, wollte das Berliner Ministerium nunmehr aktiv diesen Prozeß unterstützen und vorantreiben. Dazu schien eine Stärkung der Institutionen auf zentraler Ebene sowie eine Schwächung der Kommunalverwaltungen und der Betriebe notwendig zu sein.

Bereits Mitte September 1953 legte das Ministerium für Arbeit einen ersten Entwurf vor, der „Prinzipien“ für die Ausarbeitung einer neuen Verordnung über die Lenkung der Arbeitskräfte enthielt ${ }^{56}$. Diese inhaltliche Grobgliederung sah eine Stärkung der zentralstaatlichen Instanzen vor: Die Abdeckung des Arbeitskräftebedarfs wurde zwar als gemeinsame Aufgabe der Ministerien, Staatssekretariate, Räte der Bezirke und Kreise sowie der ihnen unterstehenden Verwaltungen und Betriebe angesehen, gleichzeitig erhielt aber die Arbeitsverwaltung ihre ursprüngliche Aufgabe als Lenkungszentrale partiell zurück. Bei ihr sollten zukünftig vierteljährlich die Bedarfs- und Überhangsmeldungen zentral erfaßt werden, was wiederum die Einführung einer detaillierten statistischen Erfassung des „Arbeitsmarktes" voraussetzte. Auch die Instrumente der Arbeitskräftelenkung sollten erweitert werden: Neben den bisherigen Aufgaben der Werbung, Ausbildung und Qualifizierung der benötigten Arbeitskräfte seien die beteiligten Akteure „,insbesondere verantwortlich für die inner- und zwischenbetriebliche Lenkung [...], für die Beschaffung von Arbeitsplätzen für solche Arbeitskräfte, die aus betrieblichen Gründen freigestellt werden müssen und für die Eingliederung der auslernenden Jugendlichen in die Produktionsbetriebe ${ }^{457}$. Die zuletzt genannten Aufgabenbereiche hatten bereits in den Jahren vor 1951 zum Tätigkeitsfeld der Arbeitsverwaltung gehört und waren in der Folgezeit zunehmend in den Hintergrund geraten. Die Rückkehr zu Methoden der Arbeitskräfteplanung und -lenkung, die Ende der vierziger Jahre eingesetzt worden waren, erfolgte aber nicht vollständig: So wurde vor allem die Wiederbelebung der Arbeitseinweisung nicht thematisiert. Diese rigide Form staatlicher Arbeitsmarktpolitik blieb somit ein Kennzeichen der ersten Nachkriegsjahre, da die SED-Führung und das Ministerium für Arbeit die negativen Folgen dieser Maßnahme für das Stimmungsbild der Bevölkerung noch gut in Erinnerung hatten. Festzuhalten bleibt des weiteren, daß sich der betriebliche Handlungsspielraum deutlich verringerte. So sah der Entwurf vom 18. September im einzelnen vor, daß die Betriebe zur Übernahme von 
Arbeitskräften, vor allem den schwer vermittelbaren, „beauflagt“ werden konnten $^{58}$; jede betriebliche Entlassung bedurfte außerdem der Zustimmung durch die zuständige Abteilung für Arbeit und Berufsausbildung59. Die Stärkung der Arbeitsverwaltung war mit einem Ansteigen der Personal- und Verwaltungskosten verbunden, da zusätzliche Sachbearbeiter eingestellt werden mußten. Das Ministerium für Arbeit rechtfertigte diese Zusatzausgaben mit dem Hinweis, daß langfristig die staatlichen Ausgaben bei der Arbeitslosen- und der Sozialfürsorgeunterstützung sinken würden ${ }^{60}$.

Es blieb zunächst jedoch bei dieser internen Bestandsaufnahme; die Planungen für eine grundlegende Revision der Verordnung vom 12. Juli 1951 wurden vom Ministerium für Arbeit vorerst nicht weiter betrieben. Ende Oktober 1953 unterrichtete die Staatliche Plankommission Arbeitsminister Macher über das Vorhaben, einen Erfahrungsaustausch mit ungarischen "Fachleuten" auf dem Gebiet der Arbeitskräftelenkung durchzuführen ${ }^{61}$. Macher begrüßte diesen Schritt grundsätzlich, wies aber gleichzeitig darauf hin, daß man in der DDR mit der „Umgruppierung“ von Arbeitskräften wenig Erfahrung gesammelt habe, „da bei uns ausnahmslos das Prinzip der Freiwilligkeit angewandt wird"62. Auch wenn sich über den Ausgang dieser bilateralen Konsultationen keinerlei Hinweise in den Akten finden lassen, so erscheint doch der gezielte Versuch einer sozialpolitischen Aussprache zwischen Experten einzelner Mitgliedsstaaten des sowjetischen Herrschaftsbereiches bemerkenswert zu sein. Eine vergleichbare Initiative entfaltete das Arbeitsministerium - in Absprache mit der SED-Führung und nach sorgfältiger Vorplanung - im Frühjahr 1955, als eine Studiendelegation in die Sowjetunion flog, um dort mit Vertretern von Ministerien, anderen staatlichen Institutionen und Gewerkschaften Fragen der Lohnpolitik und der Arbeitskräftelenkung zu erörtern ${ }^{63}$. Die Reise von 1955 stand im Zusammenhang mit der Vorbereitung des zweiten Fünfjahrplanes; auch auf anderen sozialpolitischen Feldern (z. B. Sozialversicherung) wurden solche Reisen konzipiert und durchgeführt. Auf dem Gebiet der Arbeitskräfteplanung und -lenkung sowie des Systems sozialer Sicherheit ${ }^{64}$ scheinen diese Delegationsfahrten ${ }^{65}$ keine direkten Auswirkungen auf die Entwicklung in der DDR gehabt zu haben.

Eine zeitliche Verzögerung bei der Ausarbeitung der geplanten Verordnung ergab sich auch dadurch, daß das Arbeitsministerium seit Beginn des Jahres 1954 Pläne zur Anpassung einzelner arbeitsrechtlich relevanter Gesetze an die Wirtschaftsstrafverordnung vom 23. September 1948 verfolgte ${ }^{66}$. Diese war als zentra-

58 Ebenda, S. 2 .

59 Ebenda, S. 3.

$60 \mathrm{BAB}, \mathrm{DQ} 2 / 1707$, Begründung vom 18. 9. 1953, S. 10.

${ }^{61} \mathrm{Vgl}$. BAB, DQ 2/532, Minister Macher am 15.12. 1953 an die SPK.

62 Ebenda.

$63 \mathrm{BAB}, \mathrm{DQ} 2 / 2209$, Bericht der Studiendelegation (o.D.). Teilnehmer der Delegation, die sich vom 29.4. bis 26. 5. 1955 in der UdSSR aufhielt, waren: Arbeitsminister Fritz Macher, Fritz Schellhorn (ZK-Abteilung Gewerkschaften und Sozialpolitik), Otto Lehmann (FDGB-Bundesvorstand), Helmut Bauermeister (SPK), Kurt Hohndorf (Ministerium für Maschinenbau) und Kotow (sowjetischer Berater im Ministerium für Arbeit und Berufsausbildung).

${ }^{6+} \mathrm{Vgl}$. Hoffmann, Sozialistische Rentenreform?

$65 \mathrm{Vgl}$. allgemein dazu: Kaiser, Sowjetischer Einfluß auf die ostdeutsche Politik und Verwaltung.

66 BAB, DQ 2/557, Hauptabteilungsleiterin Dr. Heinze am 11. 1. 1954 an Justizministerin Benjamin. 
les Instrument zur Neuordnung der Eigentumsverhältnisse in der SBZ/DDR verabschiedet worden ${ }^{67}$. Im einzelnen ging es nunmehr um die Übernahme von Sanktionsmaßnahmen im Arbeitsrecht, die zuvor nur in Ausführung der Wirtschaftsstrafverordnung erlassen worden waren. Darüber hinaus ging es um die Aufhebung der Verordnung über die Sicherung und den Schutz der Rechte bei Einweisungen von Arbeitskräften vom 2. Juni 1948, welche die Arbeitseinweisung geregelt hatte. Die Revision muß im Zusammenhang mit den allgemeinen Bemühungen gesehen werden, Befehle, Gesetze und Direktiven aus der unmittelbaren Besatzungszeit auf den Prüfstand zu stellen. Es kann davon ausgegangen werden, daß die Anpassung des Arbeitsrechts an die veränderte politische und wirtschaftliche Lage in der DDR mit der Führung in Moskau abgesprochen war. Dieser Prozeß hatte schon mit der DDR-Gründung begonnen und war nicht vollständig abgeschlossen worden. So war beispielsweise der SMAD-Befehl Nr. 23 vom 25. Januar 1946 über den Aufbau der Arbeitsgerichte durch die Verordnung über die Neugliederung und die Aufgaben der Arbeitsgerichte vom 30. April 1953 aufgehoben worden ${ }^{68}$. Mit dem Inkrafttreten der Verordnung über die Aufgaben der Arbeitsverwaltungen und über die Lenkung von Arbeitskräften vom 12. Juli 1951 hatten nach Einschätzung der Abteilung Arbeitskraftlenkung beim Ministerium für Arbeit die SMAD-Befehle Nr. 65 vom 15. September 1945, Nr. 153 vom 29. November 1945 sowie das Kontrollratsgesetz Nr. 3 vom 17. Januar 1946 ihre Rechtsgültigkeit verloren ${ }^{69}$. Dagegen hatte die zentrale und oben erwähnte Verordnung vom 2. Juni 1948 immer noch Bestand, welche die DDR-Regierung entsprechend einer Vorlage von Arbeitsminister Macher erst am 30. September 1954 aufhob $^{70}$. Auch wenn bereits zuvor der Richtungswechsel von der Zwangsverpflichtung hin zur Arbeitskräftewerbung de facto abgeschlossen worden war, bedeutete dieser Beschluß dennoch, daß sich die DDR nunmehr auch formalrechtlich von der rigiden Arbeitskräftelenkung der ersten Nachkriegsjahre verabschiedete. Arbeitszuweisung sollte zukünftig nur noch mit Zustimmung der Betroffenen zulässig sein. Die dabei beschlossene „Verordnung über die Aufhebung der Verordnung über die Sicherung und den Schutz der Rechte bei Einweisungen von Arbeitskräften" sicherte zugleich die Weiterzahlung von Trennungsgeldern an Arbeitskräfte zu, die freiwillig außerhalb ihres ständigen Wohnsitzes Arbeit aufgenommen hatten ${ }^{71}$. In den Genuß des Trennungsgeldes kamen außerdem die Industriearbeiter und sogenannten Industriebrigaden, die vorübergehend für die Durchführung von Pflege- und Erntearbeiten in der Landwirtschaft eingesetzt wurden.

Die Überarbeitung und Zusammenfassung der bisher geltenden arbeitsrechtlichen Bestimmungen erforderten auch eine Abstimmung mit der geplanten Neufassung des Arbeitsgesetzbuches. Offensichtlich waren sich Arbeitsministerium,

67 Vgl. Braun, Die Zentrale Kommission für Staatliche Kontrolle, S. 171-176; Wentker, Das Jahr 1948 , S. $162 \mathrm{f}$.

68 $\mathrm{BAB}, \mathrm{DQ}$ 2/689, Hausmitteilung des Ministeriums für Arbeit (Abt. Arbeitsrecht) vom 9. 8. 1954 an das Sekretariat des Ministers.

69 Ebenda, Hausmitteilung des Ministeriums für Arbeit (Abt. Arbeitskraftlenkung) vom 9. 8. 1954 an das Sekretariat des Ministers.

70 BAB, DC 20 I/3-237, Bl. 5, Protokoll der Sitzung der Regierung der DDR am 30. 9. 1954.

7 Ebenda, Bl. 104 f. Vgl. Gesetzblatt der DDR 1954, S. 828. 
Ministerrat und SED-Führung zu diesem Zeitpunkt noch nicht darüber im klaren, wie der zukünftige rechtliche Rahmen für die Arbeitskräfteplanung und -lenkung insgesamt aussehen sollte. Das erklärt möglicherweise die lang anhaltende Bearbeitung des Entwurfs vom Herbst 1953, der über den Stand einer inhaltlichen Grobgliederung nicht hinausgelangt war. So mußte das Ministerium für Arbeit selbstkritisch feststellen, daß die Vorarbeiten für die geplante Verordnung innerhalb des Ministeriums noch nicht abgeschlossen waren ${ }^{72}$. Eine Forderung stand für das Arbeitsministerium aber schon fest: die Stärkung der Arbeitsverwaltung. Diese müsse - so eine Denkschrift vom 23. April 1954 - die Möglichkeit erhalten, „unmittelbaren Einfluß auf die Lenkung wie auf die Verteilung der Arbeitskräfte zu nehmen"73. Und weiter: „Sie muß vor allem in die Lage versetzt werden, die Meldepflicht für einen bestimmten Kreis von Arbeitsuchenden wieder einführen zu können." In dem Zusammenhang wurde auch die Einführung von Strafmaßnahmen vorgeschlagen, mit denen säumige Betriebe, die den Anordnungen der Arbeitsverwaltung nicht nachkamen, belegt werden sollten. In einer internen Besprechung einigten sich Arbeitsminister Macher, sein Stellvertreter Heinicke, die Hauptabteilungsleiterin Dr. Heinze sowie der zuständige Abteilungsleiter Köhn darauf, daß grundlegende Veränderungen vorgenommen werden müßten. Die "alte bürokratische Arbeitsvermittlung" sei zwar zu Recht beseitigt worden; es sei aber verfrüht gewesen, den Arbeitsämtern „fast alle Funktionen zu einer systematischen Arbeitskraftlenkung zu entziehen "74. Neben der vorgesehenen Neufassung der Verordnung vom 12. Juli 1951 beabsichtigte das Arbeitsministerium, eine Beschlußvorlage beim Ministerrat einzureichen, welche die Betriebe anweisen sollte, berufsfremd beschäftigte Baufach- und Bauhilfsarbeiter sowie landwirtschaftliche Fachkräfte zu melden und in Zukunft nicht mehr einzustellen ${ }^{75}$.

Während das Arbeitsministerium zunächst das Ziel verfolgte, mit der geplanten Verordnung die zentralen Lenkungsinstanzen zu stärken und die Kompetenzen der Betriebe einzuschränken, wandelte sich diese Zielrichtung im Laufe der Zeit. Damit wurde letztlich der ursprüngliche Reformgedanke zunehmend verwässert. Im Spätsommer 1955 lag ein erneutes Diskussionspapier vor, in dem das Ministerium einen Spagat zwischen den beiden konträren Zielen versuchte. So sollte auf der einen Seite die "organisierende und kontrollierende Funktion" der Abteilungen für Arbeit und Berufsausbildung bei den Räten und Kreisen verstärkt werden. Auf der anderen Seite sollte das nach wie vor bestehende Prinzip, wonach die Betriebe für die Werbung und den Einsatz der Arbeitskräfte „in erster Linie und unmittelbar verantwortlich sind, unbedingt bestehen bleiben und in einigen Punkten sogar verstärkt werden“. Gleichzeitig wurde „eine klare Abgrenzung" der Aufgaben der Betriebe und der ihnen übergeordneten staatlichen Organe sowie der Arbeitsverwaltung gefordert ${ }^{76}$. Der sich daran anschließende Vorschlag einer Aufgabenabgrenzung berücksichtigte die bisher gewonnenen Erfahrungen bei der

2 BAB, DQ 2/851, Aktenvermerk Stecherts vom 1. 4. 1954.

73 Ebenda, Denkschrift des Ministeriums für Arbeit vom 23. 4. 1954, S. 4.

74 BAB, DQ 2/851, Vermerk vom 29.6. 1954 über die Besprechung, S. 1.

75 Ebenda.

76 BAB, DQ 2/1609, Vorschläge für die Verbesserung der Arbeitskräftelenkung [September 1955], S. 1 . 
Zusammenarbeit der einzelnen beteiligten Akteure überhaupt nicht. Ihm lag vielmehr die ungebrochene Gläubigkeit an die zentrale Lenkungsfähigkeit und das reibungslose Zusammenspiel der jeweiligen Verwaltungsebenen zugrunde, das sich zumindest in der Vergangenheit noch nicht bewahrheitet hatte.

Im einzelnen wurde ein dreigliedriges Modell in die Diskussion eingeführt ${ }^{77}$ : In der ersten Gruppe befand sich die Staatliche Plankommission mit ihren Verwaltungseinheiten auf zentraler und regionaler Ebene. Diese war - so die Vorstellung - verantwortlich für die Aufstellung des Arbeitskräfteplanes; die Arbeitsverwaltung erhielt die Aufgabe, „vor Bestätigung der Pläne“ Stellung zu nehmen. Zur zweiten Gruppe gehörten demzufolge die Betriebe und die Vereinigungen der volkseigenen Betriebe, die „für die Erfüllung der Pläne“ verantwortlich sein sollten. Sie hatten „insbesondere die benötigten Arbeitskräfte durch eigene Werbemaßnahmen zu gewinnen, den rationellen Einsatz der Arbeitskräfte und die volle Ausnutzung des Arbeitstages zu gewährleisten und Arbeitskräfte, die über den Plan hinaus beschäftigt werden (Überhänge), in andere Betriebe umzusetzen“. In der dritten Gruppe befanden sich die Staatliche Zentralverwaltung für Statistik und ihre Bezirks- und Kreisstellen, die „analytische Berichte“ über die Planerfüllung aufzustellen hatten. Abgesehen davon, daß die unpräzise formulierten Aufgabenbereiche bekannt waren und keine wesentlichen Veränderungen enthielten, war die Abstimmung zwischen Planerstellung, -durchführung und -kontrolle bereits während des ersten Fünfjahrplanes problematisch gewesen. Sie setzte die Bereitschaft aller Beteiligten voraus, effizient und zügig zusammenzuarbeiten und vor allem Eigeninteressen (insbesondere der Betriebe) zurückzustellen. Zur Verbesserung der Arbeitskräfteplanung und -lenkung schlug das Ministerium für Arbeit vor, ein "staatliches Organ“ zu bilden, das nicht nur den Einsatz dieses Produktionsfaktors und die Einhaltung der Pläne kontrollieren, sondern auch die „volle Ausnutzung der Arbeitszeit“ und die Fluktuationsrate überprüfen sollte ${ }^{78}$. Die Kontrolltätigkeit war auf die industriellen Großbetriebe, das Verkehrs- und Bauwesen zu begrenzen ${ }^{79}$. Es blieb allerdings offen, ob dieses neu zu schaffende Gremium dem Ministerium für Arbeit unmittelbar unterstellt werden sollte ${ }^{80}$. Überschneidungen mit Aufgaben und Zuständigkeiten der Zentralen Kommission für Staatliche Kontrolle (ZKSK) waren unausweichlich, wurden in der Denkschrift aber nicht thematisiert. Darüber hinaus waren auch andere organisatorische Fragen ungeklärt, wie z.B. die personelle Stärke dieser neuen Verwaltung. Abschließend plädierte die Denkschrift für die Aufwertung der Arbeitsbücher: Diese sollten in den Betrieben und nicht mehr bei den "Werktätigen" aufbewahrt werden und außerdem sehr viel ausführlicher als bisher Auskunft geben über den Ausbildungsweg sowie den beruflichen Werdegang jedes einzelnen ${ }^{81}$.

Die hier skizzierten Bestandteile der Denkschrift wurden innerhalb des Ministeriums für Arbeit nochmals überarbeitet, blieben aber im wesentlichen bestehen und sollten offensichtlich den Mitgliedern einer Regierungskommission vorge-

\footnotetext{
Ebenda, S. 2.

78 Ebenda, S. 3.

79 Ebenda, S. 4.

80 Ebenda, S. 5.

81 Ebenda, S. 8 f.
} 
stellt werden ${ }^{82}$. In Anlehnung an die Entwicklung in der Sowjetunion, wo seit 1946 Staatliche Inspektionen des Ministeriums für Arbeitskraftreserven zur Kontrolle über den Einsatz von Arbeitskräften bestanden, sprach sich das Ministerium für Arbeit nunmehr dafür aus, das Kontrollorgan dem eigenen Zuständigkeitsbereich direkt zu unterstellen ${ }^{83}$. Die Staatliche Plankommission kritisierte, daß der vom Arbeitsministerium vorgelegte Entwurf zu unpräzise sei: Es müsse eine klare Aufgabenabgrenzung zwischen den Abteilungen für Arbeit und Berufsausbildung auf kommunaler Ebene und den vorgeschlagenen „speziellen Organen“ vorgenommen werden ${ }^{84}$. Darüber hinaus sei bei der in Aussicht genommenen Veränderung des Arbeitsbuches der zusätzliche Verwaltungsaufwand zu berücksichtigen, der sich im Zuge der Aufwertung dieses arbeitsmarktpolitischen Instruments ergeben würde ${ }^{85}$. Um die Tätigkeit der Arbeitsverwaltung und der Industrieministerien auf dem Gebiet der Arbeitskräfteplanung und -lenkung besser zu koordinieren, war ungefähr im Frühjahr 1955 eine Regierungskommission eingesetzt worden, in der führende Vertreter der Staatlichen Plankommission, des Ministeriums für Arbeit und Berufsausbildung, der Ministerien für Schwermaschinenbau, Allgemeiner Maschinenbau, Aufbau, Leichtindustrie, Land- und Forstwirtschaft sowie des Zentralvorstandes der IG Metall versammelt waren. Den Vorsitz führte die stellvertretende SPK-Vorsitzende Dr. Grete Wittkowski. Den Vorschlag des Arbeitsministeriums, unter seiner Leitung ein „staatliches Organ zur Kontrolle des Einsatzes der Arbeitskräfte" zu bilden, hielten die Kommissionsmitglieder „übereinstimmend im Prinzip für richtig“ ${ }^{86}$. Der Minister für Arbeit und Berufsausbildung wurde beauftragt, „dem Ministerrat ein Statut zur Bestätigung vor[zu]legen, und zwar in der Weise, daß die Inspektion am 1.1.1956“ beginnen konnte. Auch der unterbreitete Gedanke, das Arbeitsbuch wieder stärker für Ziele der Arbeitskräftelenkung einzusetzen, fand Zuspruch bei der Regierungskommission. Deren Mitglieder erklärten wiederum übereinstimmend, daß das Arbeitsbuch ,während der Dauer der Beschäftigung im Betrieb abgegeben und dort aufbewahrt" werden sollte ${ }^{87}$. Gegen die beabsichtigten erweiterten Eintragungen im Arbeitsbuch wurden keine Bedenken vorgebracht, mit Ausnahme der vorgeschlagenen Eintragung über die Art der Kündigung und die Kündigungsgründe. Diese Regelung könnte - so befanden die Kommissionsmitglieder - „zu einer erheblichen Behinderung der einzelnen Werktätigen in ihrer weiteren Entwicklung führen". Da der einstellende Betrieb darüber hinaus auf solche Informationen nicht angewiesen sei, lehnte man diese vorgesehene Eintragung im Arbeitsbuch ab.

$82 \mathrm{BAB}, \mathrm{DQ} 2 / 855$, Vorschläge über die Verbesserung der Arbeitskräftelenkung vom 14. 9. 1955.

83 Ebenda, S. 10f. Das Ministerium für Arbeitskraftreserven der UdSSR wurde am 15. 3. 1946 durch die Zusammenlegung der Hauptverwaltung für Arbeitskraftreserven beim Ministerrat der UdSSR und des Komitees zur Registrierung und Verteilung der Arbeitskräfte beim Ministerrat der UdSSR gebildet. Am 15. 3. 1953 erfolgte die Auflösung des Ministeriums für Arbeitskraftreserven und die Unterstellung unter das Ministerium für Kultur. Diesen Hinweis verdanke ich Dr. Matthias Uhl.

8+ BAB, DE 1/8739, Bl. 120-122, hier Bl. 120, Notiz der Staatlichen Plankommission vom 27.9. 1955.

85 Ebenda, Bl. $120 \mathrm{f}$.

86 $\mathrm{BAB}, \mathrm{DE} 1 / 8739$, Bl. 6-10, hier Bl. 9, Protokoll über die 7. Sitzung der Regierungskommission am 28. 9. 1955.

87 Ebenda, Bl. 7. 
Aus bezirklicher Perspektive war eine Überarbeitung der Verordnung vom 12. Juli 1951 ebenfalls dringend erforderlich. Diese hatte nämlich den Landesministerien das Recht eingeräumt, die Einstellung angeworbener Arbeitskräfte von der Zustimmung der zuständigen Abteilung für Arbeit abhängig zu machen, um den Arbeitskräftebedarf der sogenannten Schwerpunktbetriebe abdecken zu können $^{88}$. Nach Auflösung der Länder im Sommer 1952 übernahmen einzelne Bezirksverwaltungen diese Regelungen und baten das Berliner Ministerium nun um eine grundsätzliche Stellungnahme in dieser offenen Rechtsfrage ${ }^{89}$. Obwohl auch in den Bezirken die Notwendigkeit gesehen wurde, Fehlentwicklungen gegenzusteuern und die bisherige Arbeitskräftelenkung einer gründlichen Revision zu unterziehen, so waren doch die daraus gezogenen Schlußfolgerungen unterschiedlich. Während sich das Ministerium für Arbeit bekanntlich für eine Stärkung der Zentralinstanzen ausgesprochen hatte, plädierten einige Bezirksverwaltungen dafür, die betrieblichen Handlungsspielräume unter Berücksichtigung der volkswirtschaftlichen Gesamtplanung zu erhalten. Demnach behielt die Arbeitsverwaltung das Recht, die Entlassung bestimmter Personengruppen (Schwerbeschädigte, VdN-Angehörige, Jugendliche) von ihrer Zustimmung abhängig zu machen. Dagegen sollte sie nicht das Recht erhalten, „die Werbemaßnahmen der Betriebe oder Arbeitsuchenden zu beschränken" $"$.

Weil Mitte der fünfziger Jahre Insuffizienzen der zentralen Arbeitskräfteplanung und -lenkung in zunehmenden Maße auftraten, auf die noch ausführlicher einzugehen sein wird, gewann ein Ziel immer mehr an Bedeutung: die Sicherung der Vollbeschäftigung. Die Entlassung von Beschäftigten im Uranbergbau, aber auch in Großbetrieben der Schwerindustrie zwang die Arbeitsverwaltung dazu, sich vorrangig mit der beruflichen Wiedereingliederung dieser Personengruppe zu befassen. Da ein regionales Ansteigen der Arbeitslosenzahlen unerwünscht war, bemühte sich das Arbeitsministerium verstärkt darum, Konzepte zur möglichst raschen Unterbringung entlassener Arbeitskräfte in Wirtschaftsbereichen mit einem entsprechenden Arbeitskräftebedarf zu entwickeln. Das hatte wiederum Folgen für die laufende Debatte über die Verbesserung der Planung und Lenkung von Arbeitskräften: Für die Planungsverwaltungen vergrößerte sich der Handlungsdruck; eine grundlegende Revision der staatlichen Arbeitskräftelenkung schien dringend geboten. Arbeitsminister Macher unterstrich bei einer internen Beratung, daß „es auf Grund der politischen und ökonomischen Entwicklung erforderlich ist, solche Maßnahmen einzuleiten, die gewährleisten, daß das Recht jedes Bürgers der Deutschen Demokratischen Republik auf Arbeit und die Versorgung

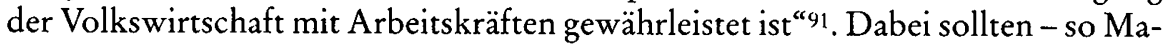

8s Vgl. 1. Durchführungsbestimmung zur VO über die Aufgaben der Arbeitskräfteverwaltungen und die Lenkung der Arbeitskräfte vom 7. 8. 1951 (\$3, Abs. 13), in: Gesetzblatt der DDR 1951, S. 753755 , hier S. 755.

89 Vgl. BAB, DQ 2/1670, Rat des Bezirkes Dresden (Abt. Arbeit und Berufsausbildung) am 5. 10. 1955 an das Ministerium für Arbcit und Berufsausbildung (Abt. Arbeitskraftlenkung).

yo BAB, DQ 2/1667, Denkschrift des Rates des Bezirkes Erfurt, am 10.3. 1956 für das Ministerium für Arbeit und Berufsausbildung, S. 7.

$91 \mathrm{BAB}, \mathrm{DE}$ 1/8766, Bl. 217-223, hier Bl. 217, Protokoll über die Beratung der zentralen Arbeitsgruppe zur Ausarbeitung von Vorschlägen für dic Verbesserung der Erfassung, Planung, Bilanzierung und Lenkung der Arbeitskräfte im Ministerium für Arbeit und Berufsausbildung am 15.3. 1956. 
cher - Vorschläge ausgearbeitet werden, die „eine exakte Erfassung aller Arbeitskräfte gewährleisten, [und] den rationellen Einsatz der Arbeitskräfte sichern". Die Abteilung Arbeitskraftlenkung beim Ministerium für Arbeit und Berufsausbildung arbeitete daraufhin einen weiteren Entwurf für eine gesetzliche Neuregelung der Arbeitskräftelenkung aus, der die Mitwirkung der Arbeitsverwaltung bei der Planaufstellung beinhaltete ${ }^{92}$, die bisher ausschließlich der Staatlichen Plankommission vorbehalten war. Ungefähr zeitgleich begann das Arbeitsministerium in Berlin damit, die jeweiligen Sozialexperten aus den Bezirksverwaltungen in die Diskussion einzubeziehen ${ }^{93}$. Dieses Angebot zur Beteiligung ging jedoch primär auf die Initiative einzelner Bezirke zurück, die sich zuvor mit Anfragen und Appellen an das Ministerium gewandt hatten.

Die Abteilung Arbeitskraftlenkung legte am 20. April 1956 eine weitere Denkschrift vor, in der die Bezirks- und Kreisverwaltungen mehr Berücksichtigung fanden. Dies sollte sich in der Einführung des "territoriale[n] Prinzip[s]“ bei der Arbeitskräfteplanung niederschlagen ${ }^{94}$. Dieser Vorschlag war nicht nur ein Entgegenkommen gegenüber den „örtlichen Organen der Staatsmacht", sondern auch eine Konsequenz der bisher gesammelten Erfahrungen: Vor allem die nach wie vor herrschende Wohnungsknappheit hatte in der Vergangenheit den zwischenund überbezirklichen Ausgleich von Arbeitskräften erheblich eingeschränkt. Da zahlreiche Beschäftigte familiär an ihren Wohnort gebunden waren, verabschiedeten sich die Staatliche Plankommission und das Arbeitsministerium immer mehr von der Vorstellung, Arbeitskräfte bedarfsgerecht und umgehend verteilen zu können. Nunmehr galt es, in unmittelbarer Nähe zu den Wohnorten Arbeitsplätze anzubieten. Als weiteres Ziel setzte sich die Abteilung des Arbeitsministeriums die Verhinderung von Arbeitslosigkeit - auch dies eine Reaktion auf alarmierende Berichte einzelner Bezirksverwaltungen. Nach den Vorstellungen des Ministeriums für Arbeit war für Anfang Juni ein Abschluß der laufenden Diskussion geplant ${ }^{95}$; erst dann sollte ein Gesetzentwurf vorgelegt werden. In der Folgezeit überarbeitete die zuständige Abteilung mehrmals den Entwurf „für eine gesetzliche Neuregelung auf dem Gebiet der Arbeitskräftelenkung“, ohne daß substantielle Veränderungen oder Ergänzungen vorgenommen wurden ${ }^{96}$.

Aus nicht erkennbaren Gründen geriet die Debatte innerhalb des Ministeriums für Arbeit und Berufsausbildung ins Stocken und wurde erst im Frühjahr 1957 fortgesetzt. Nun präsentierte das Ministerium erstmals einen Gesetzentwurf, der sich vor allem darum bemühte, die Zuständigkeiten der beteiligten „Organe“ klar

$92 \mathrm{BAB}, \mathrm{DQ} 2 / 1667$, Entwurf der Abt. Arbeitskraftlenkung vom 6. 4. 1956, S. 1.

93 So befanden sich unter den insgesamt elf Mitgliedern einer zentralen Arbeitsgruppe allein vier Vertreter aus den Bezirks- bzw. Kreisverwaltungen (Erfurt, Berlin, Halle und Plauen). Vgl. BAB, DQ 2/1667, Ministerium für Arbeit (Lieselotte Arndt) am 10.4. 1956 an die ZK-Abt. Arbeit, Sozialund Gesundheitswesen (Ramuda).

$94 \mathrm{BAB}, \mathrm{DE}$ 1/8766, Bl. 198-216, hier Bl. 204, Denkschrift der Abt. Arbeitskraftlenkung vom 20. 4. 1956.

95 Ebenda, B1. 198.

\% Vgl. BAB, DQ 2/1707, 5. Entwurf vom 14. 5. 1956; BAB, DQ 2/730, Entwurf vom 15. 5. 1956; $\mathrm{BAB}, \mathrm{DQ} 2 / 855$, Entwurf vom 19.5. 1956; $\mathrm{BAB}, \mathrm{DQ} 2 / 853$, Entwurf vom 11.6. 1956; BAB, DQ 2/1707, 8. Entwurf vom 14.6. 1956. 
voneinander zu trennen ${ }^{97}$. Dabei wurde die Verordnung vom 12. Juli 1951 jedoch nur in einzelnen Teilen überarbeitet: Nach wie vor blieben die Betriebe, in Zusammenarbeit mit den ihnen übergeordneten Organen, d.h. vor allem den VVB verantwortlich für die Bedarfsdeckung in ihrem Zuständigkeitsbereich. Das Berliner Ministerium hatte - so sah es der Entwurf weiter vor - die Aufgabe, den überbezirklichen Ausgleich von Arbeitskräften zu organisieren und zu koordinieren. Daran war die Arbeitsverwaltung Ende der vierziger bzw. Anfang der fünfziger Jahre letztlich gescheitert, was zur Folge hatte, daß dieses Aufgabengebiet fallengelassen wurde. Es überraschte, daß sich das Ministerium angesichts der bestehenden Probleme bei der Planung und Steuerung des Arbeitskräftepotentials eine "alte" Last wieder aufschulterte. Zwischen den beiden Ebenen (Betriebe, Arbeitsministerium) befanden sich die „örtlichen Räte“, denen die Aufgabe zugeteilt wurde, "die örtlichen Arbeitskräftereserven zu mobilisieren "98. In diesem Zusammenhang sollten die Kreisverwaltungen das Recht erhalten, betriebliche Werbemaßnahmen von ihrer Zustimmung abhängig zu machen. Auf diese Weise konnte der bestehende Handlungsspielraum der Betriebe spürbar reduziert werden. Dazu trug auch die vorgesehene Regelung bei, wonach die Arbeitsverwaltung einzelnen Betrieben „Einstellungsbeschränkungen“99 auferlegen durfte - eine Maßnahme zur Bekämpfung der Arbeitskräftehortung. Neu war der Vorschlag, Berufsberatungsstellen in den Stadt- und Landkreisen zu bilden, welche die Unterbringung von Jugendlichen in Lehrstellen zu unterstützen hatten ${ }^{100}$. Des weiteren hatten sie für die Absicherung des Berufsnachwuchses in allen Wirtschaftszweigen zu sorgen. Dagegen sollte das Arbeitsbuch wegfallen. Der Entwurf sah statt dessen die etappenweise Einführung eines "neuen Dokumentes" vor ${ }^{101}$. Nicht mehr aufgegriffen wurde ein zentrales Vorhaben des Arbeitsministeriums, ein nicht näher qualifiziertes „staatliches Organ“ zu errichten, das Planung und Lenkung der Arbeitskräfte überwachen sollte. In späteren Entwürfen des Frühjahrs 1956 waren aus diesem Kontrollorgan sogenannte Arbeitskräfteinspektionen geworden, die dem Arbeitsministerium direkt unterstanden. Davon war im Entwurf vom 18. März 1957 keine Rede mehr.

Der Minister für Arbeit und Berufsausbildung unterrichtete am 20. März die Vorsitzenden der Räte der Bezirke über die geplante Verordnung und bat um ihre Stellungnahmen ${ }^{102}$. In einem ausführlichen Anschreiben listete Macher die wesentlichen Beweggründe auf, die ihn und sein Ministerium dazu bewogen hatten, eine gesetzliche Neuregelung vorzunehmen. Dabei hob er besonders hervor, daß die Zuständigkeiten und Eingriffsmöglichkeiten der staatlichen Verwaltungen erweitert werden müßten. Dies kam den Bezirksverwaltungen und den dortigen Abteilungen für Arbeit und Berufsausbildung entgegen, standen sie doch in der Vergangenheit oftmals im Zentrum der Kritik über die unzureichende Arbeitskräftelenkung. Gleichzeitig wollte Macher die Gewichtsverlagerung aber so ver-

97 $\mathrm{BAB}, \mathrm{DQ} 2 / 1251$, Thesen des Ministeriums für Arbeit und Berufsausbildung zur Verordnung über die Lenkung der Arbeitskräfte vom 18. 3. 1957.

${ }_{98}$ Ebenda, S. 2 ( $\left.\$ 3\right)$.

${ }_{99}$ Ebenda, S. 5 ( $\$ 5$, Abs. 7$)$.

100 Ebenda, S. 8 f. $(\$ 11)$.

101 Ebenda, S. 9 (\$12).

$102 \mathrm{BAB}, \mathrm{DQ} 2 / 1708$. 
standen wissen, daß die betriebliche Verantwortlichkeit nicht eingeschränkt werden sollte. Um einen Rückfall in die Phase der rigiden Arbeitskräftelenkung Ende der vierziger Jahre auszuschließen, betonte er außerdem den Verzicht auf Zwangsmaßnahmen. Es war letztlich nicht weiter überraschend, als einzelne Bezirke wenige Tage später ihre prinzipielle Zustimmung zur geplanten Verordnung signalisierten ${ }^{103}$. Zahlreiche Räte der Bezirke verlangten offensichtlich eigene Sanktionsmaßnahmen (Ordnungsstrafen) gegenüber Betrieben, die den Anweisungen nicht gefolgt waren ${ }^{104}$. Auf Tagungen mit Vertretern der Bezirksverwaltungen, der Fachministerien sowie der IG Energie versuchte das Arbeitsministerium die Hoffnungen, die sich mit der in Aussicht gestellten Verordnung verbanden, etwas zu dämpfen und erinnerte an die Eigenverantwortlichkeit aller beteiligten Stellen. So erklärte etwa Hauptabteilungsleiterin Dr. Heinze am 4. Juni 1957: „Ich bitte Euch nochmals, verlaßt Euch nicht zu sehr auf diese Verordnung, denn die operative Lenkung der AK [Arbeitskräfte] ist nur eine Seite des Problems. Gute analytische Arbeit und gute Zusammenarbeit mit den HV [Hauptverwaltungen] und Betrieben und gute Einschaltung der Räte und Volksvertretungen ist dabei die andere Seite."105

Die Wiederaufnahme der Gesetzesausarbeitung war mit dem Versuch verbunden, die sozialpolitischen Akteure zusammenzubringen und dafür eine Kommission einzusetzen. Deshalb beschloß der Wirtschaftsrat auf seiner Sitzung am 17. April 1957, eine Regierungskommission „auf dem Gebiet Arbeit und Löhne einschließlich Arbeitskräftelenkung und Kaderausbildung zu bilden"106. Sie sollte Entscheidungen des Wirtschaftsrates bzw. des Ministerrates vorbereiten und die Tätigkeit der beteiligten Ministerien koordinieren. Eine der Hauptaufgaben der Regierungskommission bildete außerdem die "Ausarbeitung von Maßnahmen zur Verbesserung des Systems der Arbeitskräfteplanung, der Arbeitskräftelenkung und -werbung mit dem Ziel einer größeren Einflußnahme durch die örtlichen Staatsorgane“ ${ }^{107}$. Anfang Mai legte die zuständige Abteilung bei der Staatlichen Plankommission einen neuen Entwurf vor, welcher der gestiegenen Bedeutung der Bezirksverwaltungen Rechnung trug ${ }^{108}$. Im August brachte das Ministerium für Arbeit und Berufsausbildung einen eigenen Verordnungsentwurf in die Diskussion ein, der ebenfalls eine Erweiterung der Rechte der staatlichen Verwaltungen, vor allem der Räte der Bezirke und Kreise, gegenüber den Betrieben vorsah ${ }^{109}$. Das Ministerium für Chemische Industrie stimmte dem Entwurf grundsätzlich zu und brachte einige Änderungsvorschläge in die Diskussion ein. Dazu

${ }^{103}$ Vgl. BAB, DQ 2/1708, Vorsitzender des Rates des Bezirkes Gera am 29.4. 1957 an Minister Macher.

104 BAB, DE 1/8766, Bl. 115f., Aktennotiz (Schulz) vom 30.4. 1957.

$105 \mathrm{BAB}, \mathrm{DE} 1 / 3652, \mathrm{Bl} .86-94$, hier Bl. 94, Bericht des Ministeriums für Arbeit und Berufsausbildung vom 10.6. 1957.

106 BAB, DE 1/3625, Bl. 1 f., Notiz vom 18. 4. 1957. In der Kommission sollten vertreten sein: Staatliche Plankommission, Ministerium für Arbeit und Berufsausbildung, Ministerium der Finanzen, FDGB-Bundesvorstand, IG Metall, IG Textil, jeweils ein Ministerium „des Bereiches Schwerindustrie [...] und Maschinenbau", Ministerium für Leichtindustrie, Ministerium für Land- und Forstwirtschaft, Bezirk Leipzig, „ein Vertreter der Wissenschaft“.

10. Ebenda, Bl. 2.

${ }_{108} \mathrm{BAB}, \mathrm{DE}$ 1/3656, Bl. 1-6, 3. Entwurf der SPK vom 10.5.1957.

109 BAB, DQ 2/1708. 
gehörte vor allem der Versuch, die nach wie vor bestehende Fluktuation von Arbeitskräften wirksamer einzuschränken. Einleitend erklärte der Minister, daß vom Recht auf eine freie Arbeitsplatzwahl nicht „ein Recht auf einen ungerechtfertigten Arbeitsplatzwechsel" abgeleitet werden könnel10. In solchen Fällen sollten nach einer Neueinstellung drei Monate lang nur 85 Prozent des Tariflohnes ausbezahlt werden. Außerdem schlug das Ministerium für Chemische Industrie vor, Betrieben „mit relativ hohen Verdienstmöglichkeiten“ Einstellungen zu untersagen, die über ihren jeweiligen Arbeitskräftebedarf hinausgingen"11. Die dabei in Aussicht gestellten Instrumente zur Durchsetzung dieser Zielsetzung waren aber wenig erfolgversprechend: Sie konzentrierten sich erneut auf die Verbesserung der "Methoden zur Ermittlung des Arbeitskräftebedarfs" bzw. der Kontrolle der in den Planvorgaben enthaltenen Lohnfonds. Die Betriebe sollten somit keinerlei Handlungsspielräume erhalten, um zusätzliche Arbeitskräfte einzustellen. Abschließend sprach sich das Ministerium dafür aus, „arbeitsgeographische Karten“ anzufertigen, um die Erfassung der Arbeitskräftesituation in den einzelnen Kreisen zu optimieren ${ }^{112}$. Diese Karten, die sich ,in kleinerem Umfange in der Praxis bereits vielfach bewährt“ hätten, sollten von den „örtlichen Staatsorganen“ erstellt werden und im einzelnen die Industriezentren und ihren Belegschaftsstand, nach Wohnbereichen unterteilt, erfassen.

Während das Ministerium für Schwerindustrie mit dem Verordnungsentwurf einverstanden war ${ }^{113}$, machte das Ministerium für Handel und Versorgung erhebliche Bedenken geltend, die sich auf die Wirksamkeit der vorgeschlagenen Methoden und Instrumente bezogen. Ohne große Umschweife trug Staatssekretär Dressel die Einwände seines Ministeriums vor: „Es erscheint daher grundsätzlich zweifelhaft, ob es angebracht ist, eine Regierungsverordnung mit dieser Bezeichnung zu erlassen, in der in sehr starken Formulierungen den Ministerien und sonstigen zentralen Organen sowie den örtlichen Räten große Aufgaben der Lenkung der Arbeitskräfte übertragen sind, ohne daß die Realisierungsmöglichkeit aufgezeigt wird." 114 Diese Kritik bezog sich in erster Linie auf das Fehlen von primär wirtschaftspolitischen Instrumenten zur Steuerung des Arbeitskräftepotentials, wie z.B. die weitere Ausdifferenzierung des Lohn- und Gehaltsystems. Mit veränderten Zuständigkeiten der beteiligten Verwaltungen und entsprechender „ideologischer Unterstützung“ lasse sich eine entscheidende Verbesserung bei der Arbeitskräfteplanung und -lenkung nicht erzielen - ,jedenfalls dann nicht, wenn diese Lenkung mit den wirtschaftlichen Interessen der betreffenden Werktätigen nicht übereinstimmt “. Der Staatssekretär im Ministerium für Handel und Versorgung listete noch einige Beispiele auf, die seine Gesamteinschätzung bestätigten, und kam zum Ergebnis, daß der Verordnungsentwurf nochmals überarbeitet wer-

10 BAB, DQ 2/1708, Minister für Chemische Industrie am 5. 8. 1957 an den stellvertretenden Minister für Arbeit und Berufsausbildung Heinicke, S. 1.

111 Ebenda, S. 2.

112 Ebenda.

$113 \mathrm{BAB}, \mathrm{DQ} 2 / 1708$, Minister Apel am 6. 8. 1957 an den stellvertretenden Minister für Arbeit und Berufsausbildung Heinicke. Apcl machte einschränkend darauf aufmerksam, daß das ZK der SED auf dem 32. Plenum allgemeine strukturelle Veränderungen für die Verwaltungen angekündigt hatte, die zunächst abgewartet werden sollten.

114 Ebenda, Dressel am 12.8. 1957 an Heinicke, S. 1. 
den sollte. Das Ministerium für Kohle und Energie lehnte den Entwurf sogar kategorisch $\mathrm{ab}$ und forderte eine Ausweitung der Zuständigkeiten für die Bezirksund Kreisverwaltungen: Minister Goschütz war zwar mit der "Grundidee“, die Werbung und Einstellung von Arbeitskräften von der Genehmigung durch die „örtlichen Organe" abhängig zu machen, einverstanden ${ }^{115}$. Der Verordnungsentwurf gehe allerdings - so lautete der zentrale Kritikpunkt - ,in der Festlegung von Einzelheiten zu weit" und gebe den Bezirks- und Kreisverwaltungen zu wenig Möglichkeiten, „die durchaus notwendige Lenkung der Arbeitskräfte nach den eigenen Erfordernissen zu regeln“. Der Entwurf solle daher nochmals überarbeitet werden, wobei in der geplanten Verordnung ,nur die grundsätzlichen Rechte und Pflichten der örtlichen Organe und der Betriebe festgelegt werden sollten"116.

Den Vorschlag des Ministeriums für Handel und Versorgung, im Rahmen der Arbeitskräftelenkung die entsprechenden Anreizfunktionen zu stärken, beurteilte die zuständige Abteilung bei der Staatlichen Plankommission äußerst skeptisch. Vor allem den lohnpolitischen Maßnahmen wurde nun eine stark verminderte Wirkungskraft zugeschrieben: „Der Lohn unter volkswirtschaftlichen Gesichtspunkten hat seine Bedeutung zur Lenkung der Arbeitskräfte verloren." Dieser diente vielmehr den Betrieben dazu, Arbeitskräfte abzuwerben, so daß übergeordnete wirtschaftspolitische Ziele in den Hintergrund gerieten bzw. verdrängt wurden: „Ungesetzliche Lohnerhöhungen und unreale Normen führten dazu, daß Betriebe von volkswirtschaftlich geringer Bedeutung höhere effektive Löhne zahlen als Betriebe von großer volkswirtschaftlicher Bedeutung, so daß kaum noch Anreiz für die Arbeitskräfte besteht, in den volkswirtschaftlich wichtigsten Betrieben zu arbeiten"117. Die Reaktion verdeutlichte eine gewisse Rat- und Konzeptionslosigkeit auf seiten der zentralen Planungsbehörde, die sich jedoch auch in einem bekannten Dilemma befand. Sie sollte die betrieblichen Freiräume bei der Werbung von Arbeitskräften stärker reglementieren und einschränken, ohne aber repressive Methoden der Arbeitskräftelenkung einzusetzen. Diesen Zielkonflikt konnten die DDR-Regierung und die SED-Führung nicht auflösen. Das erklärt wiederum, daß die Ausarbeitung der Verordnung so viel Zeit in Anspruch nahm. Statt dessen mußten kurzfristig Übergangslösungen gefunden werden: So faßte der Wirtschaftsrat am 22. August 1957 einen Beschluß, der die Vorsitzenden der Räte der Bezirke und Kreise bzw. die Oberbürgermeister der Städte verpflichtete, „stärkeren Einfluß auf die Versorgung der zentralgeleiteten Betriebe mit Arbeitskräften zu nehmen" 118 . Zur Eindämmung der Arbeitskräftefluktuation erhielten die führenden Vertreter der Bezirks- und Kreisverwaltungen das Recht, den geplanten Beschäftigtenstand in einzelnen Betrieben zu reduzieren ${ }^{119}$. Für den Fall, daß sich Betriebe und kommunale Verwaltung nicht einigen konnten, die Arbeitskräftesituation vor Ort aber „für bestimmte Betriebe die volle Ausschöpfung

115 BAB, DQ 2/1708, Minister Goschütz am 10. 10. 1957 an Minister Macher, S. 1.

116 Ebenda, S. 2.

$117 \mathrm{BAB}, \mathrm{DE}$ 1/8766, Bl. 71-79, hier Bl. 72, Begründung zur Verordnung über die Lenkung der Arbeitskräfte vom 8. 8. 1957.

118 BAB, DE 1/3647, Bl. 75 f, Richtlinie zur Regelung der Arbeitskräfteversorgung in den Betrieben der sozialistischen Wirtschaft.

119 Davon ausgenommen blieben: Landwirtschaft, Baustoffindustrie, Bauwirtschaft sowie Schwerpunktbetriebe des Ministeriums für Kohle und Energie. Ebenda, Bl. 76. 
des Arbeitskräfteplanes“ nicht zuließ, hatte der zuständige Fachminister auf Antrag des Vorsitzenden des Rates des Bezirkes zu entscheiden. Zuvor hatten sich die Staatliche Plankommission und das Arbeitsministerium in dieser Angelegenheit nicht eingeschaltet, sondern die Herstellung eines Konsenses den Konfliktparteien überlassen. Darüber hinaus wurde Betrieben der sozialistischen Wirtschaft untersagt, sich gegenseitig Arbeitskräfte abzuwerben. Keiner Einstellungsbeschränkung unterlagen solche Arbeitskräfte, die im gegenseitigen Einverständnis der beteiligten Betriebe ihren Arbeitsplatz wechselten, sowie Beschäftigte aus der privaten Wirtschaft.

Trotz prinzipieller Zustimmung zur eingeführten Regelung bei den beteiligten Verwaltungen rechnete die Zentrale Kommission für Staatliche Kontrolle in der Folgezeit mit negativen Auswirkungen auf das Stimmungsbild der Bevölkerung. Zahlreiche Betriebsleiter und Funktionsträger des kommunalen Verwaltungsapparates sahen offensichtlich in der erlassenen Richtlinie des Wirtschaftsrates, die nicht veröffentlicht worden war, eine Einschränkung des in der Verfassung garantierten Rechts der Freizügigkeit und befürchteten ein Ansteigen der Westwanderung 120. Darüber hinaus registrierte die Staatliche Plankommission, was nicht weiter überraschte, Proteste auf seiten einzelner Werksleiter und Arbeitsdirektoren gegen die Einschränkung bei der Arbeitskräftewerbung. Diese „opponierten“ gegen den Beschluß, da sie „bisher mühelos von anderen Betrieben ihren Arbeitskräftebestand durch Neueinstellung infolge Abwerbung auffüllen konnten"121.

Nachdem weder das Ministerium für Arbeit und Berufsausbildung noch die Staatliche Plankommission eine Entscheidung darüber getroffen hatten, wie die zukünftige Arbeitskräftelenkung gestaltet werden sollte, ergriffen einzelne Stadtkreise die Initiative. So wies der Rat der Stadt Erfurt darauf hin, daß die privaten Betriebe in seinem Zuständigkeitsbereich besser mit Arbeitskräften versorgt wären als die volkseigenen Betriebe. Diese Entwicklung sei „restlos auszuschalten" ${ }^{122}$. Arbeitsminister Macher wurde gebeten, einem von der Stadtverwaltung erarbeiteten Aufgabenkatalog zuzustimmen, zu dem unter anderem die Verhängung einer Einstellungssperre für Privatbetriebe gehörte'23. Die Auflösung des Ministeriums für Arbeit und Berufsausbildung, dessen Zuständigkeiten auf das neu gebildete Komitee für Arbeit und Löhne sowie die Staatliche Plankommission übertragen wurden, hatte vermutlich dazu geführt, daß die Arbeiten an der geplanten Verordnung mehr als ein halbes Jahr liegen geblieben waren.

Einen Neuanfang wagte das Komitee für Arbeit und Löhne, das Vertreter der zuständigen Ministerien zu einer gemeinsamen Arbeitstagung am 26. August 1958 einlud. Dabei wurden vier Arbeitsgruppen gebildet, die jeweils eigene Aufgabenschwerpunkte erhielten ${ }^{124}$. Dort sollten in der Folgezeit Vorschläge ausgearbeitet werden, um die Fluktuation wirksam zu senken und die Arbeitsuchenden wieder

$120 \mathrm{Vgl}$. BAB, DE 1/9597, Bl. 109 f., Notiz der SPK (HA Planung der Arbeitskräfte) vom 4. 11. 1957.

121 Ebenda, Bl. 103-107, hier Bl. 104, Stellungnahme der SPK (HA Planung der Arbeitskräfte) vom 6. 12.1957.

122 BAB, DE 1/8765, Bl. 58-62, hier Bl. 58, Rat der Stadt Erfurt am 2. 7. 1958 an Minister Macher.

123 Ebenda, Bl. 61.

${ }_{124}$ BAB, DE 1/8765, Bl. 1-4, hier Bl. 1, Protokoll über die 1. Beratung der Arbeitsgruppe zur Überarbeitung der gesetzlichen Bestimmungen zur Verbesserung des Systems der Lenkung und Werbung der Arbeitskräfte am 26. 8. 1958. 
in den Arbeitsprozeß zu integrieren. Außerdem ging es darum, Vorschläge zur Verbesserung der beruflichen Nachwuchssteuerung sowie des betrieblichen Produktionsablaufes zu unterbreiten. Die vierte Arbeitsgruppe, die sich mit den innerbetrieblichen Problemen der Arbeitskräftelenkung zu beschäftigen hatte, legte im August 1958 ein erstes Konzept vor, das allerdings wenig Neues enthielt ${ }^{125}$. So wurde etwa grundsätzlich bestätigt, daß die Betriebe der sozialistischen Wirtschaft „im Rahmen ihrer wirtschaftlich-operativen Selbständigkeit für den Einsatz und die Lenkung der Arbeitskräfte verantwortlich" seien ${ }^{126}$. Dabei würde jedoch der bestätigte und mit den „örtlichen Organen“ abgestimmte Arbeitskräfteplan die wesentliche Grundlage bilden. Die Arbeitsgruppe sparte dagegen die strittigen Fragen nahezu vollständig aus: So enthielt das vorgelegte Konzeptpapier keinerlei Angaben zur betrieblichen Lohnpolitik. Vor allem dieses war in der Vergangenheit oftmals Stein des Anstoßes für die Arbeitsverwaltung gewesen, da es zahlreichen Betrieben gelungen war, mit Hilfe eigener lohnpolitischer Maßnahmen Arbeitskräfte zu gewinnen bzw. sogar aus anderen Betrieben abzuwerben. Diese Entwicklung sollte ursprünglich wieder rückgängig gemacht werden, doch dazu präsentierte die Arbeitsgruppe keine Vorschläge.

Am 19. September 1958 brachte das Komitee für Arbeit und Löhne (Sektor Rationeller Einsatz der Arbeitskräfte) ein Thesenpapier in die Diskussion ein, das sich jedoch in wesentlichen Teilen den Vorstellungen der Staatlichen Plankommission und des mittlerweile nicht mehr bestehenden Ministeriums für Arbeit und Berufsausbildung anschloß127. Ende des Monats lag dann auch ein eigener Verordnungsentwurf dieser Institution vor, der ebenfalls auf eine Stärkung der Kreisund Bezirksverwaltungen sowie eine Schwächung der Betriebe zielte ${ }^{128}$. Die Debatte drehte sich seit geraumer Zeit - darauf ist mehrfach hingewiesen worden nicht nur um den erneuten Aufbau einer eigenständigen Arbeitsverwaltung, sondern auch um die Aufgaben und Ziele einer wieder stärker zentralisierten Arbeitskräftelenkung. Dabei versuchte die Abteilung Arbeitskräfte der Staatlichen Plankommission das Aufgabenfeld abzustecken: „Das sozialistische Prinzip der Verteilung der gesellschaftlichen Arbeitskraft verlangt, daß im sozialistischen Staat weder Arbeitslosigkeit noch ein größerer ungedeckter Bedarf bestehen." ${ }^{129}$ Offen blieb allerdings die Frage, welche Funktionen die staatlichen Verwaltungen auf der regionalen und kommunalen Ebene erhalten und wie die Aufgabentrennung zu den Betrieben erfolgen sollte. Einmütigkeit herrschte nur darüber, daß die Arbeitskräftelenkung zu verbessern war, wobei alle Vorschläge eine Stärkung der zentralen Institutionen beinhalteten.

Das Komitee für Arbeit und Löhne präsentierte am 30. Dezember 1958 einen erheblich überarbeiteten Verordnungsentwurf, der inhaltlich zum Teil sehr weitreichende Bestimmungen enthielt ${ }^{130}$. Vor allem die bisher bestehenden betrieblichen Freiräume bei der Anwerbung von Arbeitskräften sollten fast vollständig

$125 \mathrm{BAB}, \mathrm{DE} 1 / 8765$, Bl. 5-8.

${ }^{126}$ Ebenda, Bl. 5.

$127 \mathrm{BAB}, \mathrm{DQ} 3 / 714$.

${ }^{128} \mathrm{BAB}, \mathrm{DQ} 3 / 714$, Entwurf vom 30. 9. 1958.

${ }^{129}$ BAB, DE 1/3510, Bl. 4-11, hier Bl. 5, Konzeption vom 2. 12. 1958.

$130 \mathrm{BAB}, \mathrm{DQ} 3 / 714$. 
aufgehoben werden. Das bedeutete zwar eine erhebliche Stärkung der Räte der Bezirke und Kreise, war aber nicht automatisch verbunden mit einer Rückkehr zu den Praktiken der Arbeitseinweisung der späten vierziger Jahre. So betonte der Entwurf ausdrücklich, das „Einverständnis der betreffenden Bürger“ sei Voraussetzung für die Arbeitskräftelenkung ${ }^{131}$. Dennoch befürchtete der stellvertretende Vorsitzende des Ministerrates Heinrich Rau, daß die geplante Verordnung eine "heftige Diskussion und auch Beunruhigung" hervorrufen würde, „da die in der Verordnung enthaltenen Bestimmungen als eine Aufhebung der Freizügigkeit, als Arbeitszwang etc. betrachtet" werden könnten. Außerdem argumentierte Rau, die Durchführung der Verordnung sei „nicht ohne einen riesigen Bürokraten-Apparat möglich“. Im Fall einer Verwirklichung würde sich rasch eine "große Diskrepanz zwischen Aufwand und Resultat ergeben" ${ }^{132}$. In der Folgezeit sollte sich die Position Raus bei der SED-Führung und beim DDR-Ministerrat durchsetzen. $\mathrm{Da}$ beide Führungsgremien einem Beschluß in dieser Frage jedoch aus dem Weg gingen, setzte sich die Diskussion endlos fort. Das Argument Raus, die Verabschiedung der Verordnung in der bisher vorliegenden Fassung gefährde die politische Legitimation der SED-Herrschaft, hatte allerdings frühzeitig Wirkung gezeigt. Auf diese Weise läßt sich auch die Hinhaltetaktik der Staats- und Parteiführung erklären. Beide erkannten zwar die Notwendigkeit, die Arbeitskräfteplanung und -lenkung zu reformieren, schreckten aber vor den von Rau in Aussicht gestellten Folgen zurück: Sie befürchteten offenbar eine Verschlechterung der Stimmungslage in der Bevölkerung und ein Ansteigen der Fluchtzahlen. Deshalb wurde die Verabschiedung der geplanten Verordnung zur Reorganisation der Arbeitskräftelenkung vorerst zurückgestellt.

Ein wesentlicher Grund für die enorm lange Entstehungsphase der geplanten Verordnung bestand außerdem in der anhaltenden Konzeptionslosigkeit der SED-Führung und der Staatlichen Plankommission. Weder das Politbüro noch das Sekretariat des ZK der SED bemühten sich um eine rasche Entscheidung. Dadurch erwuchsen wiederum begrenzte Handlungsspielräume für die nachgeordneten Ministerien und Verwaltungen. Der vom Komitee für Arbeit und Löhne eingebrachte Verordnungsentwurf stieß auch in der Folgezeit auf den Widerspruch des stellvertretenden Vorsitzenden des Ministerrates Rau, der zugleich Minister für Außenhandel und Innerdeutschen Handel war ${ }^{133}$. Dagegen signalisierten einzelne Bezirksverwaltungen ihre Zustimmung zum Entwurf: Insbesondere die Ausdehnung des Geltungsbereiches auf die Privatwirtschaft wurde besonders begrüß $t^{134}$. Rau schien jedoch mit seiner ablehnenden Stellungnahme eine Min-

131 Ebenda, $\$ 14$, Abs. 1.

132 BAB, DQ 3/714, Rau am 12. 1. 1959 an den Vorsitzenden des Komitees für Arbeit und Löhne Heinicke.

133 BAB, DE 1/8765, Bl. 21, telefonische Mitteilung vom 14. 1. 1959.

134 BAB, DQ 3/714, Rat des Bezirkes Gera (Wirtschaftsrat, Abt. Arbeit) am 15. 1. 1959 an das Komitee für Arbeit und Löhne. Ohne Abänderungswünsche stimmten die Ministerien für Gesundheitswesen, Post- und Fernmeldewesen, Auswärtige Angelegenheiten, Staatssicherheit sowic die Bezirke Rostock, Leipzig, Neubrandenburg, Frankfurt/Oder und Halle zu. Ergänzungsvorschläge bei prinzipieller Zustimmung - brachten neben der Bezirksverwaltung Gera die Ministerien für Verkehrswesen, Bauwesen, Kultur und für Inneres sowie die Bezirke Potsdam, Cottbus, Suhl, Schwerin und Dresden vor. Rau blieb dagegen bei seiner ablehnenden Haltung. BAB, DQ 3/714, Sektor Rationeller Einsatz der Arbeitskräfte am 26. 1. 1959 zur Komiteevorlage, S. 1. Das Komitee 
derheitsposition einzunehmen. So wies die Abteilung „Sektor Rationeller Einsatz der Arbeitskräfte" der Staatlichen Plankommission ausdrücklich darauf hin, daß die Auffassung Raus, die Verordnung trage wenig zur Lösung des Arbeitskräfteproblems bei, im Widerspruch zu den Einschätzungen der Bezirks- und Kreisverwaltungen stehe ${ }^{135}$. Gleichwohl kam es nicht zu einer raschen Verabschiedung durch die Führungsgremien der SED und den DDR-Ministerrat. Offensichtlich verfügte Rau, von 1950 bis 1961 Mitglied des Politbüros, über ausrechenden Einfluß innerhalb der SED-Führung, um das gemeinsame Vorhaben der Staatlichen Plankommission und des Komitees für Arbeit und Löhne hinauszuzögern. Das Komitee für Arbeit und Löhne rückte von seinen Vorstellungen kaum ab und legte am 9. Februar 1959 einen leicht überarbeiteten Verordnungsentwurf vor, der die Einwände Raus unberücksichtigt ließ136. Damit schienen sich die beiden konträren Positionen weiter zu verhärten.

In dieser Pattsituation griff die Staatliche Plankommission Vorschläge des Ministeriums für Arbeit und Berufsausbildung vom März 1957 wieder auf, Arbeitskräfteinspektionen bei den Abteilungen Arbeit der Wirtschaftsräte der Räte der Bezirke zu errichten. Sie bekamen umfassende Aufgaben „zur Sicherung der Einhaltung der Arbeitskräftepläne in Betrieben und Einrichtungen der gesamten Wirtschaft [und] zur richtigen Verteilung der Arbeitskräfte auf die einzelnen Zweige der Volkswirtschaft " ${ }^{\text {" }}$ ugeteilt ${ }^{137}$. Die Inspektionsstellen sollten pro Bezirk zwischen sechs und acht Mitarbeiter haben; innerhalb der Staatlichen Plankommission sollten ebenfalls acht Mitarbeiter neu eingestellt werden, die für die Leitung und Koordinierung zuständig waren. Erstmals wurden außerdem Fragen der Verteilung, Lenkung und Werbung von Arbeitskräften mit der Berufsberatung von Jugendlichen verknüpft ${ }^{138}$. Dadurch sollte offensichtlich die bedarfsgerechte Steuerung verbessert werden. Eine geringfügig überarbeitete Version legte die zuständige SPK-Abteilung Anfang Juni erneut vor ${ }^{139}$.

Die Staatliche Plankommission arbeitete ungefähr zeitgleich einen Verordnungsentwurf „über die Lenkung der Arbeitskräfte“ aus ${ }^{140}$. Unübersehbar war das Bestreben, die Fluktuation von Arbeitskräften zu unterbinden und dabei die bisher bestehenden Handlungsspielräume der Betriebe erheblich einzuschränken. Dazu gehörte auch das offensive Vorgehen gegen die sogenannte Hortung von Arbeitskräften in den Betrieben. So legte der Verordnungsentwurf deutlich fest, daß „jede Planerfüllung [...] mit der im Arbeitskräfteplan festgelegten Anzahl von Arbeitskräften zu erreichen" sei ${ }^{141}$. Betrieben und Verwaltungen sollte es zukünftig nicht mehr gestattet sein, Arbeitskräfte „über den Plan hinaus zu beschäftigen“. Eine Umverteilung von Arbeitskräften in andere Wirtschaftszweige „aus

für Arbeit und Löhne lehnte seinerseits die vorgebrachten Ergänzungen und Verbesserungen nahezu vollständig ab. Ebenda, S.6-9.

135 Ebenda, S. 2.

$136 \mathrm{BAB}, \mathrm{DE} 1 / 8766, \mathrm{Bl} .1-15$.

137 BAB, DE 1/8764, Bl. 321-328, hier Bl. 323, Denkschrift der Abt. Koordinierung der Planung der Arbeitskräfte vom 13.5. 1959.

138 Ebenda, Bl. $326 \mathrm{f}$.

139 BAB, DE 1/8765, Bl. 14-18, Denkschrift vom 4.6. 1959.

$140 \mathrm{BAB}, \mathrm{DE} 1 / 8744, \mathrm{Bl} .145-150$, Entwurf [Juni 1959].

${ }^{141}$ Ebenda, S. 2 (\$2, Abs. 3). 
volkswirtschaftlichen Gründen" mußte demzufolge mit den Betroffenen und den betrieblichen Gewerkschaftsorganisationen abgesprochen werden ${ }^{142}$. Es fällt insgesamt auf, daß die Bezirksverwaltungen und die Fachministerien gegenüber den Betrieben deutlich mehr Kompetenzen zugewiesen bekamen, was sich bereits in früheren Entwürfen angekündigt hatte. Der vorzeitige Abbruch des 2. Fünfjahrplanes und das Einsetzen des Siebenjahrplanes 1959 führten vermutlich dazu, daß die geplante Verordnung erneut zurückgestellt wurde. Die Staatliche Plankommission beschäftigte sich in der Folgezeit wieder mit allgemein gehaltenen Überlegungen „zur Verbesserung der Planung und Leitung der gesellschaftlichen Arbeit “ 143 und versuchte dabei die neue wirtschaftspolitische Rahmenplanung zu berücksichtigen ${ }^{144}$. In dem Zusammenhang rückte zeitweise die Bekämpfung der zwischenbetrieblichen Fluktuation in das Zentrum des Interesses: Die zuständige Abteilung der Staatlichen Plankommission arbeitete dazu einige Vorschläge aus. So sollten unter anderem Facharbeiter bei einem Arbeitsplatzwechsel nur in der Lohngruppe $\mathrm{V}$ und angelernte Arbeitskräfte höchstens in der Lohngruppe III neu eingestellt werden ${ }^{145}$. Dadurch erhoffte man sich offensichtlich eine partielle Aufhebung der Lohnanreize, die bisher eine häufige Ursache für den Betriebswechsel gewesen waren.

Die von der Staatlichen Plankommission ausgearbeiteten Vorschläge zur institutionellen Neustrukturierung sowie zur inhaltlichen Neuausrichtung der Arbeitskräftelenkung lagen der Wirtschaftskommission beim Politbüro des ZK der SED Ende Oktober 1959 vor. Die zuständige Fachabteilung der SED-Führung verlangte, daß die Vorlage nochmals überarbeitet werden sollte. Die dabei aufgeworfenen Fragen bezogen sich auf die geplante Errichtung der Arbeitskräfteinspektionen: Deren Aufgabenbereiche seien unklar formuliert worden; die Abgrenzung von anderen staatlichen Organen bleibe unklar ${ }^{146}$. Die Abteilung Arbeitskräfte bei der Staatlichen Plankommission verteidigte ihren Vorschlag und konnte zumindest erreichen, daß die Wirtschaftskommission am 21. Dezember 1959 beschloß, die Abteilung Arbeit der Bezirksverwaltung in zwei ausgewählten Bezirken (Karl-Marx-Stadt, Cottbus) personell zu verstärken ${ }^{147}$. Mit diesem Beschluß waren jedoch keine Arbeitskräfteinspektionen geschaffen worden; statt dessen blieb es bei der bisherigen Verwaltungsstruktur. Die neu einzustellenden Verwaltungsangestellten sollten offenbar die den Inspektionsstellen zugedachten Aufgaben wahrnehmen. Darüber hinaus sollten in den beiden genannten Bezirken Arbeitsbrigaden gebildet werden, die von der Staatlichen Plankommission und dem Komitee für Arbeit und Löhne geleitet wurden und in denen Vertreter der kommunalen Verwaltung und der Massenorganisationen saßen ${ }^{148}$. Diese kamen

${ }_{142}$ Ebenda $(\$ 3)$.

${ }^{143}$ BAB, DE 1/8765, Bl. 174-180, Entwurf der SPK-Abt. Arbeitskräfte vom 25. 8. 1959.

${ }^{1+4}$ Ebenda, Bl. 177. Eine überarbeitetc Fassung dieser Denkschrift ging am 27.8. 1959 an den SPKVorsitzenden Leuschner. Vgl. BAB, DE 1/11839, Bl. 1-8.

$145 \mathrm{BAB}, \mathrm{DE}$ 1/8764, Bl. 350-352, hier Bl. 351, Vorschläge für Maßnahmen zur Einschränkung der Fluktuation der Arbeitskräfte (o.D.).

146 Ebenda, Bl. 445-448, hier Bl. 445 f., Leiter der SPK-Abt. Arbeitskräfte (Duscheck) am 26. 11. 1959 an den SPK-Vorsitzenden Leuschner.

1+7 BAB, DE 1/8767, Bl. 21, Vorsitzender des Komitees für Arbeit und Löhne (Heinicke) am 5. 1. 1960 an den stellvertretenden Minister der Finanzen (Geiss).

${ }^{1+8}$ Ebenda, Bl. 16-18, Arbcitsrichtlinie vom 5. 2. 1960. 
aber oftmals der Aufforderung, sich an den Arbeitsgruppen zu beteiligen, nicht nach ${ }^{149}$. Es war vorgesehen, eine neue Vorlage für das ZK der SED bis Mitte Juli 1960 zu erstellen, die auf den bis dahin gesammelten Erfahrungen der Pilotstudie in den beiden Bezirken basieren sollte ${ }^{150}$.

Die Testphase in den Bezirken Cottbus und Karl-Marx-Stadt war zeitlich nicht befristet und zog sich bis in den Herbst 1960 hinein. Eine mangelhafte Vorbereitung trug mit dazu bei, daß ein frühzeitiger Abschluß nicht erreicht wurde. Die Abgrenzung der Tätigkeitsfelder der neu einzustellenden Verwaltungsangestellten blieb lange Zeit umstritten. Die Staatliche Plankommission, das Komitee für Arbeit und Löhne, die ZK-Abteilung Planung und Finanzen und die zuständigen Bezirksverwaltungen konnten sich zunächst nicht einig werden. Die Vorschläge des Instituts für Arbeitsökonomik und Arbeitsschutzforschung, das an den Beratungen beteiligt war, wurden von den anderen Akteuren nicht angenommen. Letztlich sollten die neuen Planstelleninhaber die Tätigkeit der Abteilung Arbeit beim Wirtschaftsrat der beiden Bezirken nur "qualitativ" verstärken; eine exakte Aufgabentrennung konnte dagegen nicht durchgesetzt werden ${ }^{151}$. Bei der Besprechung am 22. April setzten die Vertreter der Staatlichen Plankommission durch, daß das oberste Ziel der Arbeitskräftelenkung die Einhaltung der Pläne sein müsse. Im Zusammenhang mit der Kollektivierung der Landwirtschaft hatten offensichtlich zahlreiche Landwirtschaftliche Produktionsgenossenschaften zusätzliche Arbeitskräfte angefordert. Da die Umgestaltung der Landwirtschaft und die damit zusammenhängenden Sonderprogramme zu diesem Zeitpunkt höchste Priorität genossen, sollte nach Ansicht der Staatlichen Plankommission die Bedarfsdeckung „zu Lasten anderer staatlicher Aufgaben gehen“152. Von dieser Regelung blieben nur die neu errichteten Wirtschaftsstandorte ausgenommen, wie z.B. das Braunkohleveredelungswerk „Schwarze Pumpe“. Für die übrigen Wirtschaftszweige wurde somit die Gefährdung der im Siebenjahrplan vorgegebenen Produktionsziele billigend in Kauf genommen. Erstaunlicherweise waren diese möglichen negativen Auswirkungen nicht einmal Gegenstand der Besprechung. Die Staatliche Plankommission wollte unter allen Umständen die betriebliche Werbung von Arbeitskräften drastisch einschränken. Deshalb lehnte sie einen weiteren Vorschlag des Instituts für Arbeitsökonomik und Arbeitsschutzforschung ab, die Betriebe zu unterstützen, die eine Produktion neu aufnahmen bzw. diese erweiterten, und dazu die Pressewerbung durch den Rat des Kreises zuzulassen ${ }^{153}$. Das Verbot von öffentlicher Arbeitskräftewerbung blieb somit uneingeschränkt bestehen.

Nachdem die Studien in den Bezirken Karl-Marx-Stadt und Cottbus beendet worden waren, lag ein Konzept „zur Verbesserung der Leitungstätigkeit der staatlichen Organe auf dem Gebiet der Arbeitskräfte" Mitte September 1960 endlich vor ${ }^{154}$. Wesentliche Strukturmerkmale dieses Papiers waren zum einen die stär-

\footnotetext{
${ }^{1+9}$ Ebenda, Bl. 12-14, hier Bl. 12, Aktennotiz über eine Aussprache bei der SPK-Abt. Arbeitskräfte am 25. 3. 1960.

150 Ebenda, Bl. 1-4, hier Bl. 3 f., Bericht der SPK-Abt. Arbeitskräfte vom 7. 4. 1960.

151 BAB, DE 1/8767, Bl. 9-11, hier Bl. 9, Aktenvermerk über eine Aussprache am 22. 4. 1960.

152 Ebenda, Bl. 10.

153 Ebenda, Bl. $10 \mathrm{f}$.

154 BAB, DE $1 / 11839, \mathrm{Bl} .17-42$
} 
kere Einbettung der Betriebe in die staatliche Arbeitskräftelenkung, was letztlich auf eine Begrenzung der betrieblichen Handlungsspielräume hinauslief. In dem Zusammenhang sollte nicht nur die Position der Staatlichen Plankommission innerhalb des Verwaltungsgefüges weiter ausgebaut, sondern auch die Bedeutung der bisher eher vernachlässigten „örtlichen Organe des Staates“ erheblich verstärkt werden. Zum anderen wurden Grundlinien der bisherigen Arbeitskräftelenkung fortgeführt: Das betraf etwa die Bestätigung des Rechts auf Arbeit sowie die "freie Wahl des Berufes und die Freizügigkeit jedes Bürgers"155. Letzteres schloß vor allem die Rückkehr zu Methoden der Zwangseinweisung aus. Während der ursprüngliche Plan, Arbeitskräfteinspektionen zu bilden, endgültig fallengelassen wurde, rückte die personelle Verstärkung der „örtlichen Organe“ in den Mittelpunkt des Interesses. Die zuständigen Abteilungen der 15 Bezirksverwaltungen sollten nach den Vorstellungen der Staatlichen Plankommission insgesamt 145 zusätzliche Planstellen erhalten ${ }^{156}$.

Die Ausarbeitung der Verordnung zog sich ebenfalls in die Länge: Anfang Januar 1961 lag ein Entwurf der Staatlichen Plankommission vor, der sich an die bisher erstellten Konzeptionen eng anlehnte ${ }^{157}$. Einen Monat später schaltete sich die SED-Führung wieder ein und bestätigte die von Paul Verner vorgestellte Vorlage ${ }^{158}$. Damit ging eine inhaltliche Schwerpunktverlagerung einher: Um der gewachsenen Bedeutung der Bezirksverwaltungen gerecht zu werden, sprach sich das Politbüro dafür aus, Ämter für Arbeit und Berufsberatung zu bilden. Die Berufsausbildung wurde zu einem wichtigen Element der Arbeitskräftelenkung aufgewertet. Die vorausschauende Planung des beruflichen Nachwuchses war jedoch grundsätzlich nicht neu und wurde stellenweise auch bei der Diskussion über die inhaltliche Neubestimmung der Arbeitskräftelenkung thematisiert. Mit der Errichtung der Ämter sollte zunächst in den Bezirken Halle, Leipzig, Berlin, Frankfurt/Oder und Potsdam begonnen werden ${ }^{159}$. Gleichzeitig beschloß das Politbüro, das Arbeitsbuch und den Ausweis für die Sozialversicherung zu einem einheitlichen Dokument zusammenzufassen ${ }^{160}$. Das Politbüro beschloß schließlich am 6. Juni 1961 die Umwandlung der Abteilungen für Arbeit bei den Kreisen und Bezirken in Ämter für Arbeit und Berufsberatung. Diese Entscheidung sollte "streng vertraulich behandelt" und zunächst nicht veröffentlicht werden ${ }^{161}$. Die Verabschiedung des Entwurfs durch den Ministerrat erfolgte am 24. August ${ }^{162}$. Kurz zuvor hatte ein Ereignis erhebliche Auswirkungen auf die Arbeitskräftelenkung in der DDR entfaltet: Durch den Mauerbau am 13. August 1961 änderte sich

155 Ebenda, Bl. 18.

156 Ebenda, B1. 41.

157 BAB, DE 1/8766, B1.226-232, Verordnung zur Verbesserung der Arbeitskräftelenkung.

158 SAPMO, DY 30/J IV 2/2/748, Bl. 4 und Bl. 59-77, Protokoll der Sitzung des Politbüros am 7. 2. 1961.

159 Ebenda, Bl. 60.

160 Ebenda, Bl. 66. Ein erster Entwurf lag bereits im September 1958 vom Komitee für Arbeit und Löhne (Sektor Rationcller Einsatz der Arbeitskräftc) vor. BAB, DE 1/9058, Bl. 104-113.

161 SAPMO, DY 30/J IV 2/2/766, Bl. 4 und Bl. 49-70. Ein redigiertes Exemplar der Verordnung sollte offensichtlich an das Präsidium des ZK der KPdSU direkt gesendet werden. SAPMO, DY 30/IV 2/2A/824.

$162 \mathrm{BAB}, \mathrm{DC} 20 \mathrm{I} / 3-346, \mathrm{Bl} .3$ und Bl. 6-14. Die Verordnung wurde am 28. 8. 1961 im Gesetzblatt veröffentlicht. Gesetzblatt der DDR 1961, Teil II, S. 347-349. 
schlagartig die Situation auf dem ostdeutschen Arbeitsmarkt; die ungehinderte und unkontrollierbare Abwanderung von Menschen im erwerbsfähigen Alter wurde radikal gestoppt. Diese Maßnahme leitete auch bei der Arbeitskräftelenkung eine neue Phase ein.

Der Beschluß, Ämter für Arbeit und Berufsberatung in den Bezirken und Kreisen aufzubauen, hob die Verordnung vom 12. Juli 1951 auf und bedeutete zum Teil eine Wiederherstellung der Arbeitsverwaltung, wie sie bis 1951 bestanden hatte. Diese Aufwertung zeigte, daß auch in einer Zentralverwaltungswirtschaft nicht völlig auf eine eigenständige Arbeitsverwaltung verzichtet werden konnte. Gleichwohl kam es nicht zu einer vollständigen Wiederherstellung der Verwaltungsstrukturen, die im Frühjahr 1962 im übrigen auch noch nicht abgeschlossen war163: Zum einen wurde die Arbeitskräftelenkung mit der Berufsberatung und -lenkung verbunden. Zum anderen existierte nach wie vor kein Ministerium für Arbeit an der Spitze der Verwaltung, dessen Aufgaben von der Staatlichen Plankommission und dem Komitee für Arbeit und Löhne übernommen worden waren. Die Verordnung vom 24. August 1961 beendete eine jahrelange Diskussion, die von SED, Arbeitsministerium, Komitee für Arbeit und Löhne sowie Staatlicher Plankommission geführt worden war. Dabei ging es um die Frage, inwieweit das Arbeitsrecht durch repressive Instrumente der Arbeitskräftelenkung zu ergänzen war. Während das Komitee für Arbeit und Löhne für die bedingte Wiedereinführung der Arbeitseinweisung plädierte, sprach sich die Staatliche Plankommission strikt dagegen aus und konnte sich mit dieser Position letztlich durchsetzen. Entscheidend waren die Erfahrungen, welche die staatlichen Verwaltungen in der zweiten Hälfte der vierziger Jahre hatten sammeln können, als die Zwangseinweisungen in den Uranbergbau zu einer erheblichen Verschlechterung der allgemeinen Stimmungslage geführt hatten. Aus dem drohenden Legitimationsverfall hatten deshalb seinerzeit SED und Arbeitsministerium die nötigen Schlußfolgerungen gezogen: Anstelle der repressiv wirkenden Maßnahmen wurden immer häufiger Instrumente eingesetzt, die Anreizfunktion besaßen und letztlich eine, wenn auch begrenzte, Rückkehr zu marktregulierenden Maßnahmen bedeuteten. Die Schwierigkeiten der bedarfsgerechten Arbeitskräftelenkung, die im Verlauf der fünfziger Jahre verstärkt aufgetreten waren, überzeugten die politisch Verantwortlichen letztlich nicht davon, diesen Anfang der fünfziger Jahre eingeschlagenen Weg zu verlassen. Statt dessen wurde der Versuch unternommen, die Arbeitskräftelenkung institutionell mit der Berufsberatung zu verbinden.

163 BAB, DC 20 I/4-551, Bl. 4 und Bl. 50-56, Protokoll der Sitzung des Präsidiums des Ministerrates am 26. 4. 1962. Auf dieser Sitzung verabschiedete das Präsidium des Ministerrates einen Beschluß zur weiteren Durchführung der Verordnung zur Verbesserung der Arbeitskräftelenkung und Berufsberatung vom 24.8 .1961 . 